\title{
Dilatancy and Precursory Bulging Along Incipient Fracture Zones in Uniaxially Compressed Westerly Granite
}

\author{
Hsi-Ping LiU
}

Seismological Laboratory and Division of Engineering and Applied Sciences California Institute of Technology, Pasadena, California 91125

\author{
Alexander C. R. Livanos
}

Division of Engineering and Applied Sciences, California Institute of Technology, Pasadena, California 91125

\begin{abstract}
The existence of precursory bulge along an incipient fracture zone in a uniaxially compressed Westerly granite sample has been investigated by two optical methods. The first method is the method of slit diffraction. The cross section of a cylindrical rock sample is monitored by bringing two straightedges next to the rock sample to form two slits (each slit being formed between one straightedge and one side of the rock sample) and by illuminating alternately the two slits with a collimated laser beam. The Fraunhofer diffraction pattern is recorded on film in the direction perpendicular to the straightedge and can be interpreted as the absolute value squared of the one-dimensional spatial Fourier transform of the slit under certain conditions, thereby providing a simple method of magnification of the rock surface geometry. The conditions under which the Fraunhofer diffraction pattern can be interpreted as the absolute value squared of a one-dimensional Fourier transform are related to the radius of the rock sample, width of the slit, position of the recording film plane, and nature of deformation of the rock surface and are presented in this paper. The films are digitized by a microdensitometer. The data are analyzed by digital filtering and interpolation techniques to give a strain resolution of $10^{-5}$. During a test with the slit diffraction method, strain inhomogeneities in terms of local bulges indicative of incipient failure zones were found to develop at $\sim 92 \%$ of the uniaxial compressive strength, and their propagation is traced at 2.66-s intervals until failure. Local strains in the incipient failure zones are of the order of $10^{-2}$ before failure takes place. Because of the large amplitude of the strain inhomogeneity prior to failure recorded by the slit diffraction method, we then tried the faster method of recording without magnification by a motion picture camera. In the second test a precursory bulge in the middle of the sample first appeared at $\sim 3.75 \mathrm{~s}$ prior to failure at a load of $>99.7 \%$ of the uniaxial compressive strength. The bulge developed rapidly in successive frames until eventually a failure plane passed through this sharp bulge. The results from both tests demonstrate the formation of a concentrated weak zone as a result of the interaction and coalescence among the microcracks in the final stage of the test, which then develop into fracture zones. The bulging is the result of accentuated deformation in the weak zone because of its reduced deformation moduli. It is considered that the local bulge and orientation of the fracture zones in the first test were controlled by the stress concentration at the sample-load block interface, whereas those in the second test were controlled mainly by the inhomogeneity in the material properties within the sample. The precursor times of both tests do not fit into the empirical relationship between precursor time and fault dimension as derived from earthquakes and mine rock bursts. The precursor times of these tests are too long by 3 orders of magnitude in comparison with those given by the empirical relationship.
\end{abstract}

\section{INTRODUCTION}

The concept and the term dilatancy in granular materials were first introduced by Reynolds [1901]. He defined dilatancy to be the change in volume of a granular mass as its shape is being altered. He performed experiments with sand, among other granular materials, and recognized that both increase (positive dilatancy) and decrease in volume (negative dilatancy) are possible as a consequence of an externally imposed change in shape, depending on the initial porosity of the granular mass.

More recently, Bridgman [1949], Matsushima [1960a, b], Handin et al. [1963], and Brace et al. [1966] investigated volume change in rocks under uniaxial and triaxial test conditions, and the term dilatancy has now come to mean the change of volume relative to linearly isotropic elastic changes caused by deformation. With the exception of the Berea sandstone, the Marianna limestone, and the Repetto siltstone (all of them sedimentary rocks with high initial porosity), tested by Handin et al. [1963] under high effective confining pressure $(>0.5 \mathrm{kbar}$ for Berea sandstone and Marianna sandstone and $>0.25$ kbar for Repetto siltstone) all test results on rocks showed positive dilatancy. In particular, positive dilatancy in Westerly granite tested by Brace et al. [1966] persists to $8 \mathrm{kbar}$, the highest confining pressure in their experiments, and the

Copyright @ 1976 by the American Geophysical Union. final volume prior to failure is greater than the initial volume up to a confining pressure of $4.11 \mathrm{kbar}$. Brace et al. [1966] attributed the positive dilatant behavior to crack opening, and Scholz [1968a] correlated the amount of dilatancy with microcracking frequency. A host of dilatancy-related phenomena were also measured in the laboratory under uniaxial and triaxial loading conditions. For example, Matsushima [1960c] measured the elastic wave velocity variations in granite associated with dilatancy, Brace and Orange [1968] measured electrical resistivity in a variety of crystalline rocks undergoing positive dilatancy, and Brace and Martin [1968] measured the effect of dilatancy on pore pressure in some crystalline rocks and observed the effect of dilatancy hardening.

It is thought recently that positive rock dilatancy associated with microcrack opening could explain the reported seismic variation prior to earthquakes [Nur, 1972; Whitcomb et al., 1973; Anderson and Whitcomb, 1973; Aggarwal et al., 1973], and as a consequence, rock dilatancy is considered to be of central importance in the understanding of precursory phenomena associated with earthquakes. When it is viewed in this light, Scholz et al. [1973] describe the phenomenon of positive dilatancy in rocks as a physical basis for such possible earthquake precursors as change in seismic velocity ratio, electrical resistivity, radon emission, and geodetic measurements, Beaumont and Berger [1974] computed the effect of dilatancy on the tidal response of the crust, and Whitcomb [1975] examined 
quantitatively the dependence of vertical geodetic (including leveling, tilt, and geometric measurement relative to a celestial frame of reference), and gravity measurements in relation to dilatancy density change. A volume of crust undergoing positive dilatancy is assumed in these calculations, and the consequences are then computed. It is apparent that the extent of the precursory phenomena computed from these models depends on the degree of positive rock dilatancy measured in the laboratory.

However, the strain gauge method employed by Matsushima [1960a, b] and Brace et al. [1966] is subject to some uncertainties. In particular, the strain gauges, both axial and circumferential, can only be mounted at discrete points on the rock sample surface, and an assumption must be made concerning the strain distribution of the whole sample in order to compute the volume strain. The usual assumption is that the strain is uniform and the sample is isotropic. The volume strain $\Delta V / V_{0}$ is therefore axial strain $\epsilon_{z}$ plus 2 times the circumferential strain $\epsilon_{\theta}$,

$$
\Delta V / V_{0}=\epsilon_{z}+2 \epsilon_{\theta}
$$

This is an unsatisfactory assumption if the compliance of sample ends and loading platens is not properly matched. It becomes a poor assumption even for properly matched end conditions if weak zones form before failure and cause the strain to become inhomogeneous. Brace et al. [1966] reported that the total dilation at fracture, observed in their experiments by the strain gauge method and using assumption (1), varies at least by a factor of 2 , suggesting the possibility that the strain becomes highly inhomogeneous at high stress levels.

It is therefore important to have a method which would allow a continuous surface strain mapping of the rock sample. Such a method not only would examine the uniformity of the loading stress at the ends of the rock sample but could also trace the development of inhomogeneous strains prior to failure if the time resolution of the method were short in comparison with the characteristic time of any such development.

The study of the fracturing process in a rock sample under uniaxial and triaxial loading conditions is also important in its own right. Although the behavior of a homogeneous rock sample under relatively simple loading conditions is quite dissimilar to that of a geological structure with inclusions, largescale joints, and faults and is subject to more complicated boundary stresses, the fracture process of a rock sample may serve as a prototype for the more complicated fracture process in the crust and may help to provide a qualitative understanding for shallow earthquakes.

The direct volumeter methods used by Bridgman [1949],
Handin et al. [1963], and Brace and Orange [1968] for dilatancy measurement have low sensitivity and cannot measure any local strains. We therefore chose optics as a means for continuous strain mapping. The first optical method we used is the method of slit diffraction.

\section{Slit Diffraction Method: Theoretical Background and EXPERIMENTAL ARrangement}

Slit diffraction method. It is well known that Sommerfeld's diffraction theory integral [e.g., Pearson et al., 1969] used in conjunction with some of Kirchhoff's approximations yields accurate results if the aperture is large in comparison with a wavelength and the diffracted field is observed far from the aperture [Silver, 1962; Wolf and Marchand, 1964; Marchand and Wolf, 1966]. Figure I shows the Fraunhofer diffraction pattern of a slit formed by two parallel razor blade edges and illuminated by a collimated laser beam. The intensity distribution of the slit diffraction pattern is shown in Figure 2. The intensity across the diffraction pattern $I\left(x_{0}\right)$ is given by [ Goodman, 1968]

$$
I\left(x_{0}\right)=\frac{I_{0} l_{x}^{2}}{\lambda^{2} z^{2}} \frac{\sin ^{2}\left[\pi\left(l_{x} x_{0} / \lambda z\right)\right]}{\left[\pi\left(l_{x} x_{0} / \lambda z\right)\right]^{2}} \equiv \frac{I_{0} l_{x}^{2}}{\lambda^{2} z^{2}} \operatorname{sinc}^{2}\left(\frac{l_{x} x_{0}}{\lambda z}\right)
$$

where $\operatorname{sinc}(x)=[\sin (\pi x) / \pi x]$ is the sinc function. Mathematically, the amplitude of the diffracted light is also proportional to the one-dimensional spatial Fourier transform of the slit. The width between the two first intensity minima is given by

$$
\Delta x_{0}=2\left(\lambda z / l_{x}\right)
$$

where in (2) and (3), $\lambda$ is the light wavelength, $z$ the distance between the slit and the film plane, $l_{x}$ the width of the aperture, $x_{0}$ the distance in the direction perpendicular to the diffraction pattern and measured from center of the diffraction pattern, and $I_{0}$ the incident light intensity. The separation $\Delta x_{0}$ in (3) is inversely proportional to the slit width. Therefore the narrower the slit width, the wider the diffraction pattern. This is the magnification effect which we will use to map the surface strain of the rock sample.

The slit in the present experiment is formed between a straightedge and one side of the cylindrical rock sample. Rice [1953] showed that a rounded cylinder can be considered as a knife-edge in applying the scalar diffraction theory if the observation angle deviates less than

$$
\left(\frac{1}{4}\right) \cdot\left(\frac{\text { wavelength }}{\text { radius of curvature of cylinder }}\right)^{1 / 3}
$$

from the wave vector of the incident plane wave, and Barnett and Harris [1962] showed that the results are also independent

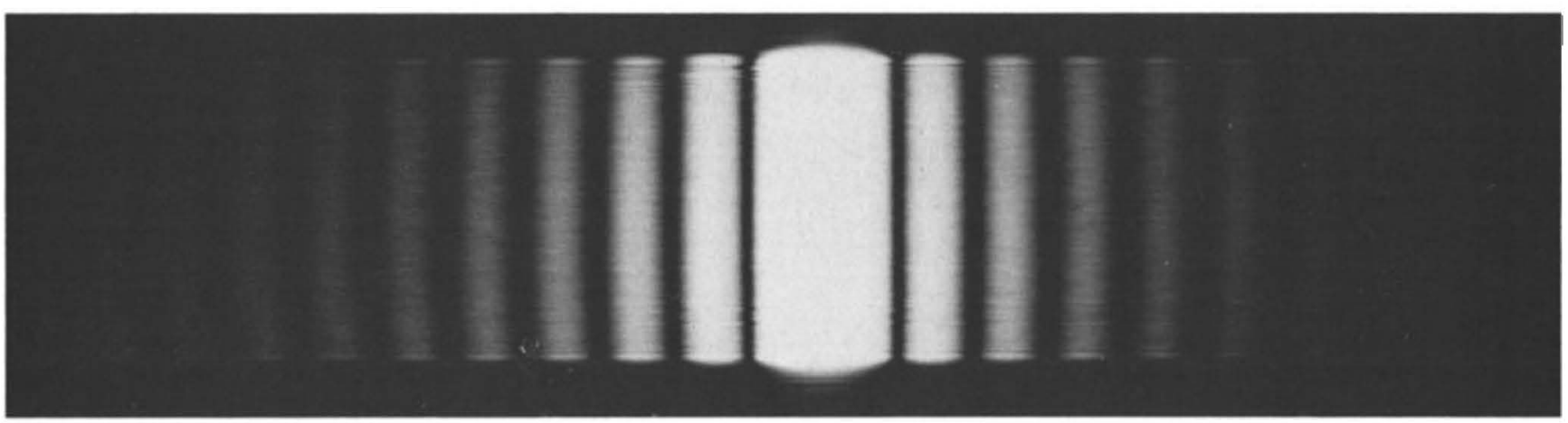

Fig. 1. Fraunhofer diffraction pattern of a finite length slit formed by two razor blades and illuminated by a collimated laser beam. 
of electrical conductivity of the material forming the diffraction edge if the above condition is satisifed. In the present experiment the quantity

$$
\begin{aligned}
\left(\frac{1}{4}\right) \cdot\left(\frac{\text { wavelength }}{\text { radius of curvature of cylindrical rock samp }}\right. & \\
= & \left(\frac{1}{4}\right) \cdot\left(\frac{0.6328 \times 10^{-4} \times 2}{0.9657 \times 2.54}\right)^{1 / 3} \\
= & 9.31 \times 10^{-3} \mathrm{rad}
\end{aligned}
$$

Since, for the present experiment, the distance $\Delta x_{0}$ is less than $3.5 \mathrm{~cm}$ in all of the diffraction patterns and $z=211.8 \mathrm{~cm}$, the maximum observation angle is $(3.5 / 2 \times 211.8)$ radians $=8.26$ $\times 10^{-3} \mathrm{rad}$. Rice's condition is therefore satisfied.

Equations (2) and (3) apply to the case of an infinite slit of constant width. The question naturally arises: when one side of a finite length slit is slightly deformed, will one still be able to use the one-dimensional diffraction formula along the slit? Or equivalently, what are the çonditions such that (2) and (3) are good approximations if $l_{x}$ varies along the slit? This question can be answered by the scalar diffraction theory and the method of stationary phase. The amplitude of diffracted light in the film plane in the Fresnel approximation is given by [e.g., Goodman, 1968]

$$
\begin{aligned}
& U\left(x_{0}, y_{0}\right)=\frac{e^{i k z}}{i \lambda z} \iint_{-\infty}^{+\infty} \mathcal{u}\left(x_{1}, y_{1}\right) \\
& \cdot \exp \left\{i \frac{k}{2 z}\left[\left(x_{0}-x_{1}\right)^{2}+\left(y_{0}-y_{1}\right)^{2}\right]\right\} d x_{1} d y_{1}
\end{aligned}
$$

where $e^{i k z}$ is the incident plane wave and $U\left(x_{1}, y_{1}\right)$ is the slit transmission function given in Figure 3.

Assume that the width of the slit is such that the Fraunhofer approximation is valid in the $x_{0}$ direction; i.e.,

$$
z \gg k\left(x_{1}^{2}\right)_{\max } / 2
$$

Equation (5) becomes

$$
\begin{aligned}
& U\left(x_{0}, y_{0}\right)=\frac{e^{i k z}}{i \lambda z} \exp \left[i \frac{k}{2 z} x_{0}^{2}\right] \int_{-a / 2}^{+o / 2} d y_{1} \cdot \int_{0}^{f\left(y_{1}\right)} \\
& \exp \left[-\frac{i k}{z} x_{0} x_{1}\right] \cdot \exp \left[i \frac{k}{2 z}\left(y_{1}-y_{0}\right)^{2}\right] d x_{1} \\
&=i \frac{e^{i k z}}{\lambda z} \exp \left[i \frac{k}{2 z} x_{0}^{2}\right] \cdot \int_{-a / 2}^{+a / 2} f\left(y_{1}\right) \\
& \operatorname{sinc}\left(\frac{f\left(y_{1}\right) x_{0}}{\lambda z}\right) \cdot \exp \left[i \frac{k}{2 z}\left(y_{1}-y_{0}\right)^{2}\right] d y_{1}
\end{aligned}
$$

The integral on the right-hand side of (7) can be evaluated by the method of stationary phase [e.g., Carrier et al., 1966]. With the assumptions (see the appendix for details of derivation)

$$
\begin{gathered}
\left|f^{\prime}\left(y_{1}\right) \frac{x_{0}}{2}\right| \leq \frac{a}{N} \quad-\frac{a}{2}<y_{1}<\frac{a}{2} \\
-a\left(\frac{1}{2}+\frac{1}{N}\right)<y_{0}<a\left(\frac{1}{2}+\frac{1}{N}\right)
\end{gathered}
$$

where $N$ is a large number and

$$
\left|f^{\prime \prime}\left(y_{0}\right) x_{0} / 2\right| \ll 1
$$

equation (7) is evaluated to give the intensity distribution

$$
\begin{aligned}
& I\left(x_{0}, y_{0}\right)=\left\{\frac{I_{0} f\left(y_{0}\right)^{2}}{\lambda^{2} z^{2}} \operatorname{sinc}^{2}\left(\frac{f\left(y_{0}\right) x_{0}}{\lambda z}\right)\right\} \\
& .\left\{\left(\frac{\pi z}{k}\right)\left|C\left(\eta_{2}\right)-C\left(\eta_{1}\right)+i\left[S\left(\eta_{2}\right)-S\left(\eta_{1}\right)\right]\right|^{2}\right\}
\end{aligned}
$$

where

$$
C(\eta)=\int_{0}^{\eta} \cos \left(\frac{\pi t^{2}}{2}\right) d t \quad S(\eta)=\int_{0}^{\eta} \sin \left(\frac{\pi t^{2}}{2}\right) d t
$$

are the Fresnel integrals and $\eta_{1}=-(k / \pi z)^{1 / 2}\left[(a / 2)+y_{0}\right], \eta_{2}=$ $(k / \pi z)^{1 / 2}\left[(a / 2)-y_{0}\right]$.

Comparing (10) with (2), we see that (10) means that the diffraction pattern can be interpreted as the absolute value squared of a one-dimensional Fourier transform of the slit in the $x_{0}$ direction but intensity modulated in the $y_{0}$ direction by the Fresnel diffraction pattern due to a slit of width $a$.

For an illustrative example pertinent to the present problem, consider the function

$$
f\left(y_{1}\right)=l_{0}+\alpha \sin \left(2 \pi y_{1} / \Lambda\right)
$$

i.e., the slit deviates from a straight slit by a sine function perturbation of amplitude $\alpha$ and wavelength $\Lambda$. Equation (8) is satisfied if

$$
(\alpha \pi / \Lambda) x_{0} \leq(a / N)=150 \times 10^{-4} \mathrm{~cm}
$$

Here the value $a / N$ is chosen to be $150 \mu \mathrm{m}$, a typical film digitizing interval along the rock axis in the present experiment. Equation (9) is satisifed if

$$
\left(x_{0} / 2\right) \alpha(2 \pi / \Lambda)^{2} \ll 1
$$

Take $x_{0}=0.75 \mathrm{~cm}$, also typically the maximum half distance sampled on the film in the present experiment; then (12) and (13) imply that

$$
\begin{gathered}
\alpha / \Lambda \leq 6.4 \times 10^{-\mathrm{s}} \\
\Lambda \gg 0.4 \mathrm{~mm}
\end{gathered}
$$

Conditions (14) and (15) give the maximum amplitude to wavelength ratio and the minimum wavelength of a sinusoidal perturbation on a straight slit such that the diffraction pattern in the present experiment can safely be interpreted by the formula (10). These conditions are satisfied in the present experiment.

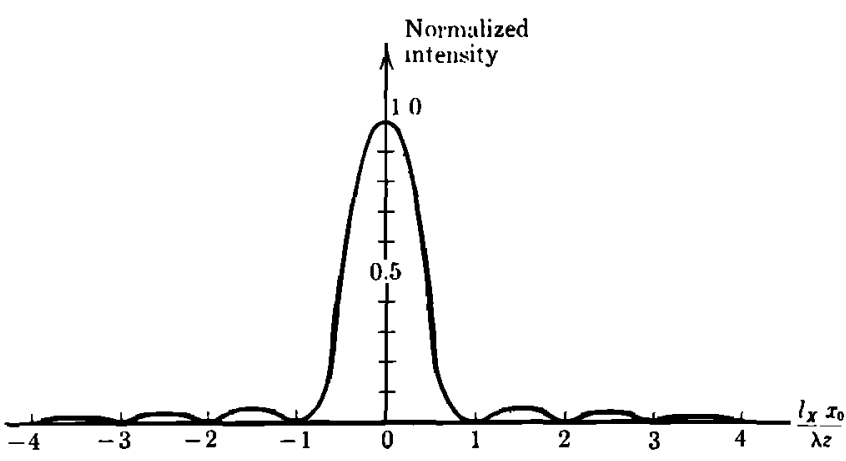

Fig. 2. Normalized intensity distribution of the slit Fraunhofer diffraction pattern. Here, $l_{x}$ is the slit width, $\lambda$ the light wavelength, $x_{0}$ the distance across the diffraction pattern, and $z$ the distance between the slit and the film plane. 


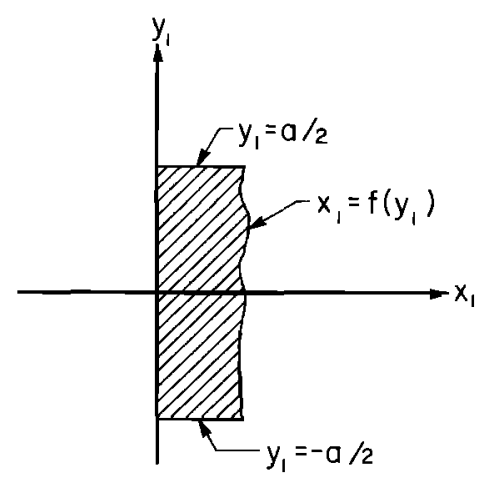

Fig. 3. Geometry of a finite, generalized one-dimensional slit. The transmission equals 1 inside the shaded area showing the slit and is 0 otherwise.

One should note, however, that (8) and (9) are only the necessary conditions such that $(10)$ is applicable to the present experiment. It is entirely possible that $(10)$ could still be valid under more general conditions. This point will be discussed further in the part on data analysis in the next section.

Experimental arrangement. The experimental arrangement is illustrated in Figure 4. The rock sample is a Westerly granite cylinder. Its dimensions are $2.4529 \pm 0.0003 \mathrm{~cm}$ in diameter and $5.0470 \mathrm{~cm}$ in length. The ends are lapped flat to 1 wavelength (sodium light), and their parallelism is better than $0.0003 \mathrm{~cm}$. The rock sample is loaded by two hardened beryllium copper loading blocks with dimensions $2.4493 \pm 0.0005$ $\mathrm{cm}$ in diameter and $4.6050 \mathrm{~cm}$ in length. The ends are also lapped flat to 1 wavelength and parallel to better than 0.0003 $\mathrm{cm}$. The reason for choosing beryllium copper for loading block material is to better match the compliance between rock sample and end loading blocks. Beryllium has a Poisson's ratio to Young's modulus ratio of $0.237 \mathrm{Mbar}^{-1}$ in comparison with $\sim 0.160 \mathrm{Mbar}^{-1}$ for most steel alloys [Oberg and Jones, 1962]. This same Poisson's ratio to Young's modulus ratio for Westerly granite varies from $0.236 \mathrm{Mbar}^{-1}[\mathrm{Fai}, 1961]$ and 0.384 $\mathrm{Mbar}^{-1}$ [Knopoff. 1954] from dynamic measurements to 0.821 , 0.961 , and $1.549 \mathrm{Mbar}^{-1}$ if we adopt the static compressibility data of Simmons and Brace [1965] and a Poisson's ratio of 0.271 [Knopoff, 1954; also consistent with the uniaxial compression $\epsilon_{\theta} / \epsilon_{2}$ data by Brace et al., 1966]. The spherical seat is used to reduce bending moments on the rock sample. The assembly is loaded by a $136,077-\mathrm{kg}(300,000 \mathrm{lb})$ capacity Baldwin universal testing machine. The load is measured by a Bourdon tube gage, which is calibrated by a strain gage load cell. Two straightedges are brought alongside the rock sample to form two slits. Each slit is formed between one straightedge and one side of the rock cylinder. The sample straightedge assembly is illuminated by a collimated helium-neon laser beam about $8 \mathrm{~cm}$ in diameter. The two razor blades, protruding $0.762 \pm 0.003 \mathrm{~mm}$ from the loading blocks, cut off any high spatial frequency corner diffraction effects at the sample-loading block interface. The two slits are blocked off alternately such that only one slit is illuminated at a time, while the diffraction pattern is recorded on $70-\mathrm{mm}$ film mounted in an automatic film transport. The frame rate of the film transport is $2.66 \mathrm{~s}$ per frame. The film plane is oriented perpendicular to the incident laser beam to $0.002 \mathrm{rad}$, and the distance between the film plane and the slits is $211.76 \mathrm{~cm}$. The distance between the two straightedges is fixed. The width of the rock cross section in the plane of the straightedges along the rock cylinder axis is therefore the separation of the two straightedges minus the sum of the widths of the two slits.

\section{Data, Data Analysis, and Results From the Slit Diffraction Method}

Data. The load-time history is shown in Figure 5. Failure occurred at $11,416 \mathrm{~kg}$ (2.37-kbar stress). Each circle signifies the time when the diffraction patterns of both slits are taken consecutively $2.66 \mathrm{~s}$ apart. The dashed line signifies the time interval when continuous shooting at a frame rate of $2.66 \mathrm{~s}$ per frame takes place. A total of 206 frames are taken before failure takes place. Figure 6 shows a series of diffraction patterns of the two slits at different load levels. Figures $6 a$ and $6 b$ show the diffraction patterns of the two slits at a load of $\mathbf{4 4 7 . 7}$ $\mathrm{kg}$ (0.09-kbar stress). Figures $6 c$ and $6 d$ are the corresponding ones at $3,134 \mathrm{~kg}(0.65$-kbar stress). Figures $6 e$ and $6 f$ show the diffraction patterns at a load of $6,715 \mathrm{~kg}$ (1.39-kbar stress). The diffraction patterns have by now become visibly wider, signifying the narrowing of the two slits (therefore an expansion of the rock sample cross section). Figures $6 g$ and $6 h$ are the data at $9,849-\mathrm{kg}$ load (2.04-kbar stress). Figure $6 \mathrm{~g}$ indicates a slight bulge at the bottom of the rock sample in the left-hand slit viewed from the direction of film transport. Figure $6 h$ indicates slight bulging at the top of the rock sample in the right-hand slit viewed from the direction of film transport. Figures $6 i$ and $6 j$ correspond to a load of $10,744 \mathrm{~kg}(2.23-\mathrm{kbar}$ stress), and they show pronounced bulging at the two diagonally opposite corners of the rock sample (bottom of Figure $6 i$ and top of Figure $6 j$ ). In addition, Figure $6 j$ indicates bulging at bottom of rock sample as well. Figures $6 k$ and $6 l$ are data at $11,048-k g$ load (2.29-kbar stress). In Figure $6 l$ we see that the top bulge in the right-hand slit (viewed from the direction of film transport) has nearly touched the straightedge from the fact that the width of the diffraction pattern central lobe has become very large at one end. Figure 7 consists of 10 slit diffraction patterns taken in the $27 \mathrm{~s}$ prior to the failure of the rock sample. In Figure $7 c$ we see that the bulged zone in the left-hand slit (viewed from the direction of film transport) has also touched the straightedge (widely spreading diffraction pattern at bottom of Figure $7 c$ ). Figure $7 g$ portrays some dynamic fracturing in progress. Figure $7 i$ shows how the slit looks at less than $6 \mathrm{~s}$ before failure, and we see that one bulged zone has advanced well over half the length of the rock sample. It shows a sharp boundary at the front of the bulged zone. Compare Figures $7 i$ and $7 g$. The slit width has increased; i.e., the rock sample cross section has shrunk ahead of the bulged zone just prior to failure. The failure load is $11,416 \mathrm{~kg}$, corresponding to a uniaxial compressive strength of 2.37 kbar. Figures $8 a$ and $8 b$ show the rock sample as retrieved after the experiment. Figure $8 a$ shows the side of the rock sample facing

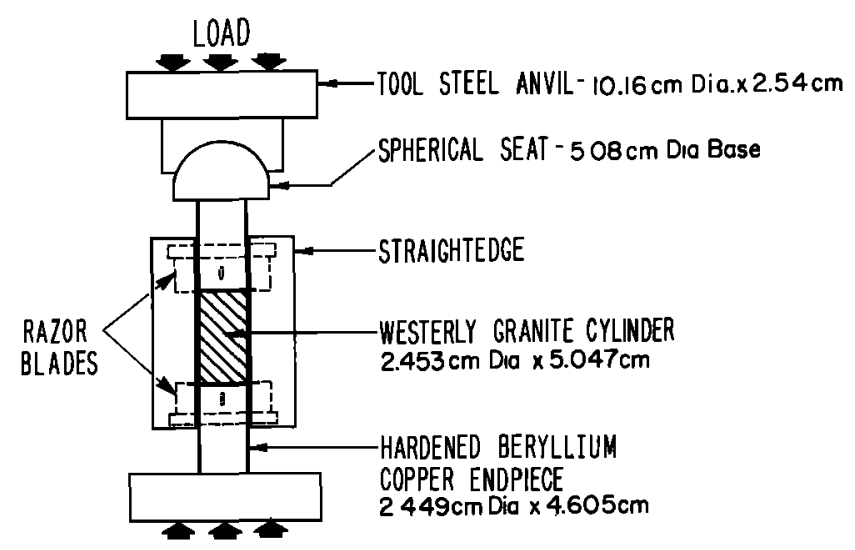

Fig. 4. Experimental arrangement of the slit diffraction method of mapping rock sample surface deformation. 


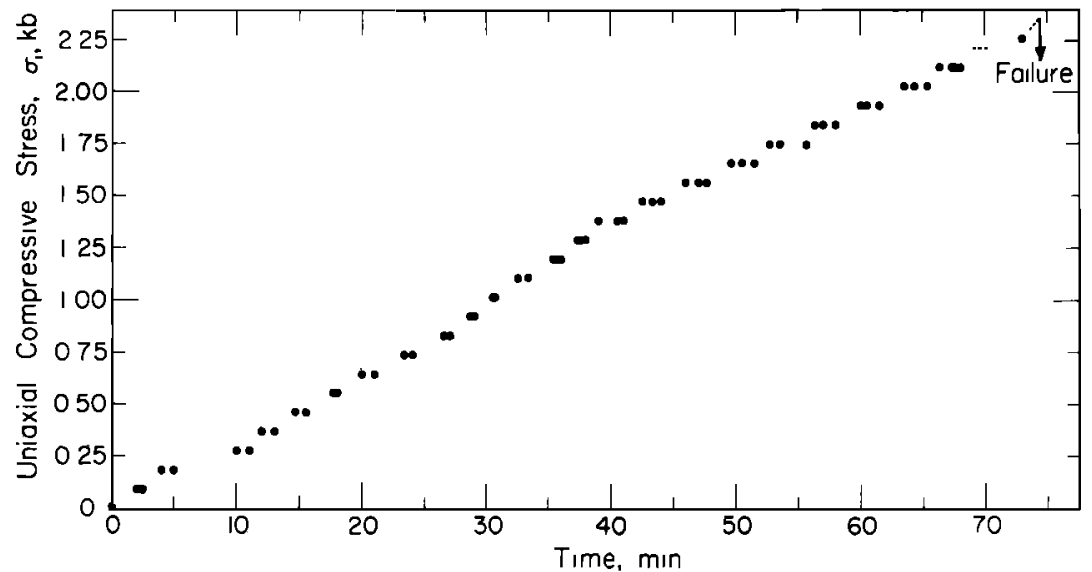

Fig. 5. Load-time history of the uniaxial compression test using slit diffraction method to map the rock sample surface deformation. Each dot signifies the time when the diffraction patterns of both slits are taken consecutively $2.66 \mathrm{~s}$ apart. The dashed lines signify the time interval when continuous shooting at a frame rate of $2.66 \mathrm{~s}$ per frame takes place.

toward the incident laser beam. The streak of Dykem layout paint indicated by the arrow in Figure $8 a$ was painted on the rock sample before the experiment roughly halfway between the two slits. This streak of paint allowed us to identify the fault orientation with respect to the position of the slits. It is seen from Figure $8 a$ that the fault zone is roughly perpendicular to the plane containing the two slits. Figure $8 b$ shows the side of the rock sample facing toward the film transport. The surface of the fault zone has a soft and flaky texture in comparison with the rock sample before the test. The relationship between the surface features on the retrieved rock sample and the diffraction patterns presented in Figures 6 and 7 will be discussed in the part on results in the present section.

Data analysis. The films were digitized by a Perkin-Elmer PDS microdensitometer data acquisition system. The films were aligned so that one cross hair of the microdensitometer was made parallel to the Fresnel diffraction patterns (see (10)) due to the two razor blades attached to the end loading blocks (see Figure 4). This can easily be done to an accuracy better than $1^{\circ}$ of arc. The digitizing interval was $50 \mu \mathrm{m}$ across the diffraction pattern. The top and bottom edges of the slit were located by the Fresnel diffraction pattern (see (10)) and according to Pearson et al. [1969]. The distance along the rock axis is then divided into 255 equal digitizing intervals. When bulging at the ends rendered it impractical to locate the top and bottom edges of the slit as defined by the two razor blades by this method (see Figure 7), the shadow of a metal piece in the film transport was used as a reference to locate the previously determined position of the top and bottom edges of the slit. The number of points digitized along the rock axis is 256 , with the exception of the two films shown in Figures $7 \mathrm{~g}$ and $7 i$, in which case the number is 128 . The position accuracy and repeatability of the Perkin-Elmer microdensitometer is $\pm 1 \mu \mathrm{m}$.

The numerical data for each film consist therefore of $\mathbf{2 5 6}$ rows of numbers (or 128 rows for film shown in Figures $7 g$ and 7i). The aim is to locate the two first-order intensity minima and from (3) to compute the slit width along the rock axis. We fit the intensity distribution by the square of a sinc function (see (2)). Figure 9 shows a typical theoretical fit near the first intensity minimum. The minima of intensity distribution as predicted by the theoretical fit and by observation coincide to within $\pm 1 \mu \mathrm{m}$, which is the repeatability and accuracy of the digitizing microdensitometer. The closeness in the fit to the square of a sinc function and the general satisfaction of (14) and (15) justify the interpretation by (10) for the first-order diffraction patterns. Previously, we remarked that (14) and (15), or equivalently, (8) and (9), are conservative in estimating the range of validity of the interpretation of slit diffraction pattern according to (10). Part of the reason is that the diffracted light due to higher-frequency perturbations on a straight slit appear mostly in higher-order diffraction fringes. This is evident from the moiré-like interference patterns in Figures 6 and 7. Therefore the interpretation of first-order diffraction patterns according to (10) is perhaps valid beyond the condition imposed by (8) and (9). We suggest the theoretical fit of intensity near the first intensity minima by the square of a sinc function to be a better criterion for the applicability of the one-dimensional Fourier transform interpretation of (10).

It is, however, relatively expensive to fit all the rows of numbers by the square of a sinc function. Instead, we used a parabolic fit in general and used the square of a sinc function fit for eight randomly selected rows for each film. The separation between the minima as predicted by the sinc function fit and as predicted by the parabolic fit is taken as a measure of the uncertainty in the slit width determination. This results in a typical strain resolution of $10^{-5}$. The slit widths at 256 points (or 128 points in film shown in Figures $7 \mathrm{~g}$ and $7 i$ ) along the rock axis in each film are determined by (3). We then take the slit widths at all these locations along the rock axis and, in accordance with (15), digitally filter out all features that are less than $1 \mathrm{~mm}$ in dimension.

Results. The slit widths as deduced by this procedure are shown in Figures 10, 11, and 13. We see in Figure 10 that the minor features of the slits are preserved up to a load of at least $3581.4 \mathrm{~kg}(0.74-\mathrm{kbar}$ stress). Figure 11 shows the slits under a load of $10,744 \mathrm{~kg}(2.23-\mathrm{kbar}$ stress) at the onset of extensive bulging. Since

$$
\Delta d_{r}=-\Delta d_{s}
$$

before the onset of local strain inhomogeneity, where $d_{r}$ is the width of the rock sample cross section and $d_{s}$ is the combined average slit width of the two slits, the lateral strain $\epsilon_{l}$ defined as the change in the width of the rock sample cross section is given by

$$
\epsilon_{l}=\frac{\Delta d_{r}}{d_{r 0}}=-\frac{\Delta d_{s}}{d_{r 0}}=\frac{-d_{1}+d_{s 0}}{d_{r 0}}
$$

where $d_{r 0}$ and $d_{s 0}$ are the initial values of $d_{r}$ and $d_{s}$, respectively. The $\epsilon_{l}$ is equal to the fractional change in diameter if there is 

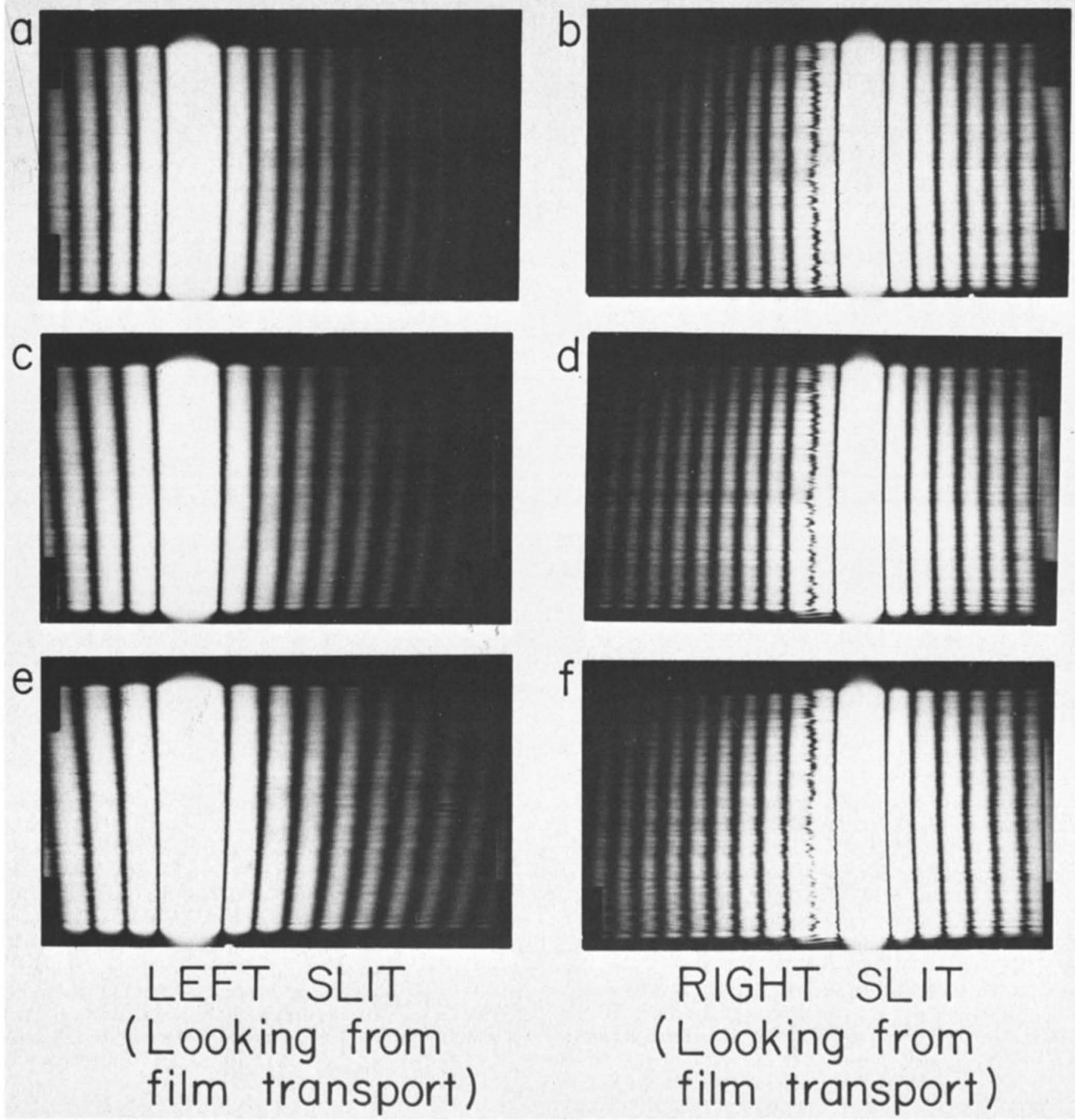

Fig. 6. A series of diffraction patterns of the two slits at different load levels. $(a, b) 447.7 \mathrm{~kg}(0.09-\mathrm{kbar}$ stress $)$; $(c, d)$ $3,134 \mathrm{~kg}$ (0.65-kbar stress); (e, $f) 6,715 \mathrm{~kg}$ (1.39-kbar stress); $(g, h) 9,849 \mathrm{~kg}$ (2.04-kbar stress); $(i, j) 10,744 \mathrm{~kg}(2.23$-kbar stress); $(k, l) 11,048 \mathrm{~kg}(2.29-\mathrm{kbar}$ stress). Arrow indicates appearance of local bulging in the rock sample cross section.

no azimuthal variation of the lateral strain. The lateral strain as a function of stress is plotted in Figure 12. The lateral strain-stress curve shows the three regions of initial crack closure, of the linearly elastic behavior, and of the positive dilatancy as observed by Brace et al. [1966]. Figure 13 shows a continuous series of plots of one slit. The slit belongs to the left side of the rock sample as shown in Figure $8 b$. It shows the progress of the bulged zone up the rock. Figure 14 shows the average slit width ahead of the bulged zone as a function of time of the slit shown in Figure 13. The increase in slit width just prior to rock failure is an indication of strain relaxation ahead of the bulged zone. This happens when the spread of the bulged zone was accelerating toward failure, which is borne out by the position of the front of the bulged zone as a function of time, shown also in Figure 14. The straightness of the diffraction fringes ahead of the bulged zone and the slit width data on the opposite side of the rock as shown in Figure 14 preclude rigid rotation or translation of the sample as a whole as the cause of the increase in slit width just prior to failure.

The slit diffraction method maps only the surface strain of a cross section of the rock sample. We need therefore to examine the retrieved rock sample fragments and infer the processes leading to fracture with the help of information obtained from the slit diffraction patterns. Figure $8 b$ shows the rock sample viewed from the direction of the film transport. The fracture 

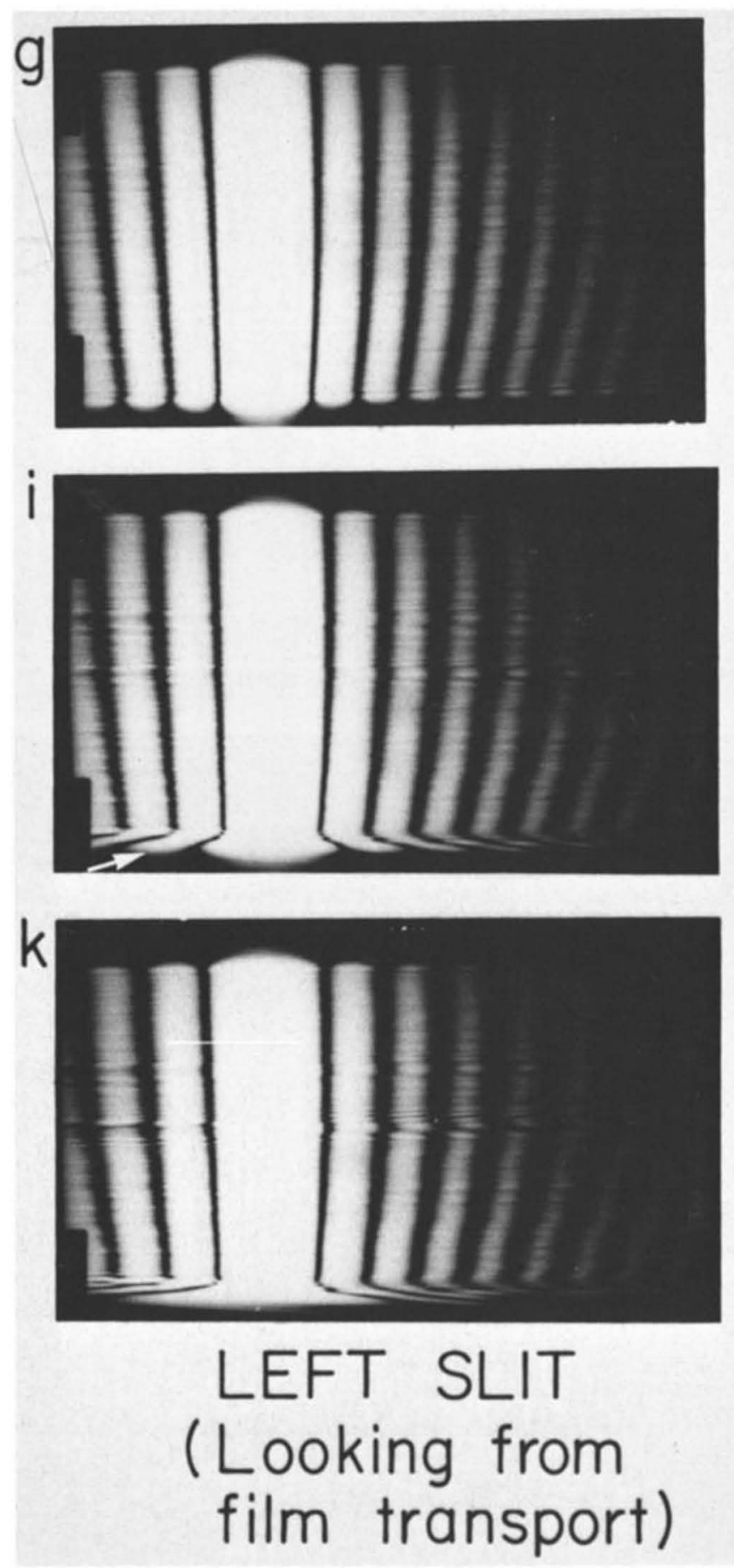
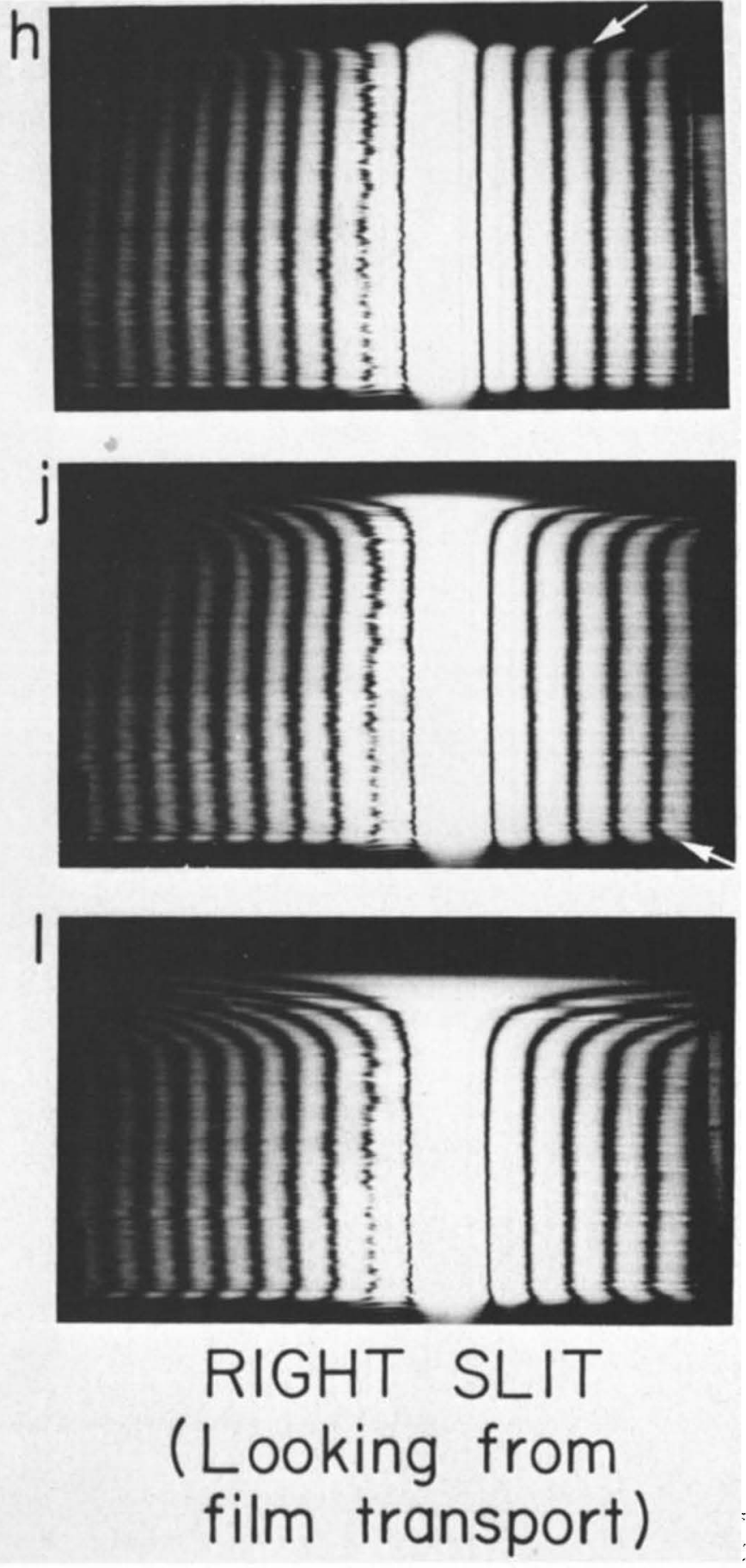

Fig. 6. (continued)

zone lies in a plane roughly perpendicular to the plane containing the two slits. The bulging observed in the diffraction patterns as shown in Figures 6 and 7 can be associated with the features on the retrieved rock samples in Figure $8 b$ in the following manner. (1) The bulging initiated at the bottom of Figure $6 i$ and extending continuously toward the top in Figures $6 k, 7 a, 7 c, 7 e, 7 g$, and $7 i$ corresponds to the weakening of the large missing zone on the left-hand side of the rock sample as shown in Figure $8 b$. The sharp boundary ahead of the bulged zone in the diffraction pattern as shown in Figure $7 i$ corresponds to the step clearly visible at the upper left corner of the retrieved rock sample as shown in Figure $8 b$. (2) The bulging, initiated at the top of Figure $6 h$, becoming pro- nounced in Figure $6 j$, and touching the straightedge in Figures $6 l, 7 b, 7 d, 7 f, 7 h$, and $7 j$, corresponds to the weakening of the shallow 'spalled' zone in the upper right corner of the retrieved rock sample as shown in Figure $8 b$. (3) The slight bulge, starting at the bottom of Figure $6 j$ and becoming more pronounced in Figures $6 l, 7 b, 7 d$, and $7 f$, corresponds to the weakening of the zone at lower right corner of the rock sample as shown in Figure $8 b$. This zone is aligned with the fracture plane.

The local bulges as deduced from the diffraction patterns in the present test have these in common: first, they are smooth, coherent surface expressions at least from the first appearance of the bulge to the stage when the bulges touched the straight- 

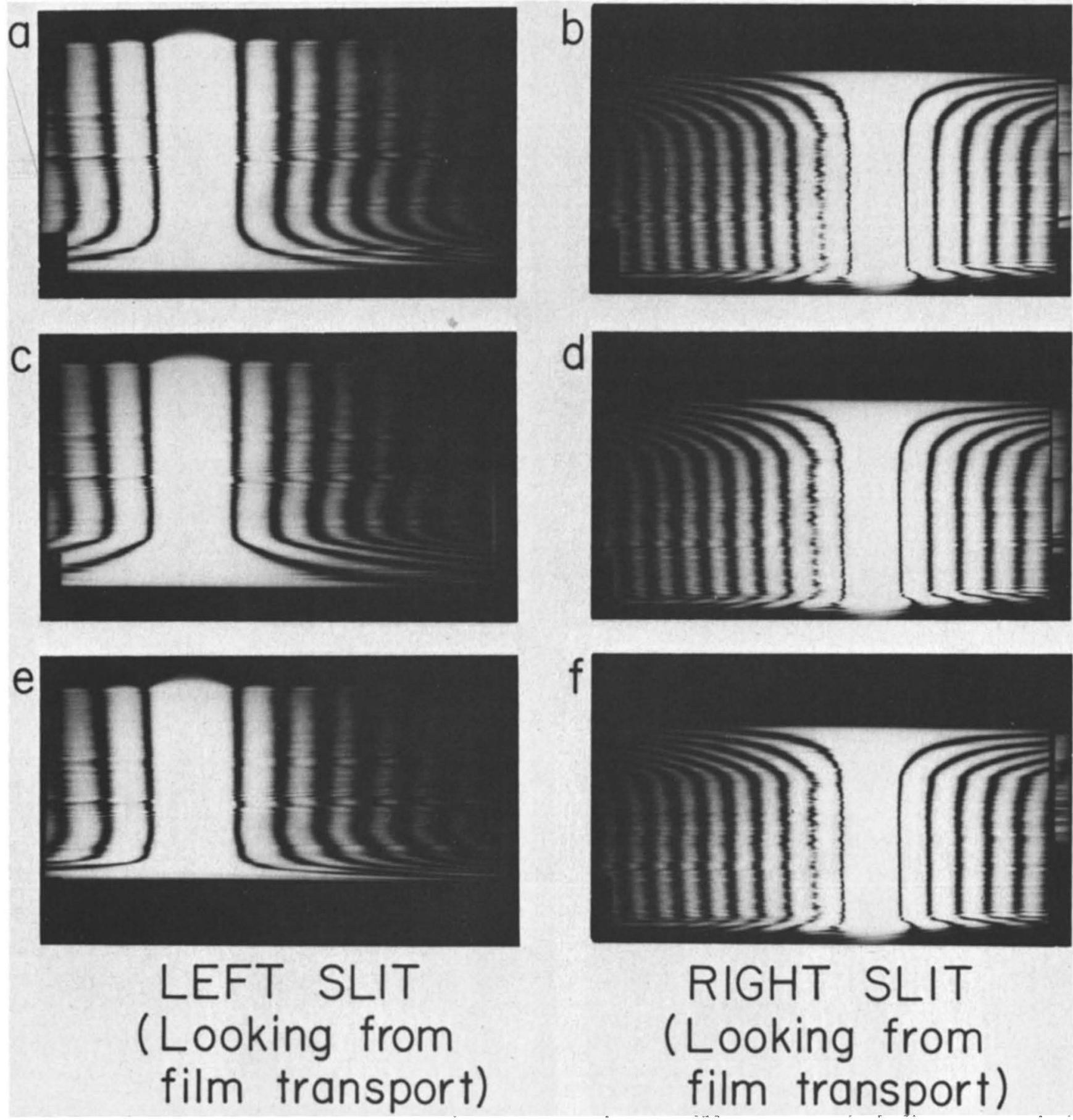

Fig. 7. Ten slit diffraction patterns taken in the $27 \mathrm{~s}$ prior to rock sample failure.

edge, and second, they all appeared at $\sim 92 \%$ of the uniaxial compressive strength. Furthermore, the bulged zonęs were weak in comparison with the original intact rock sample. That the bulged zones were weak can be substantiated by the fact that when the bulged zone touched the straightedge it did not push the straightedge away. This can be judged from the consistently straight fringe pattern ahead of the bulged zone as shown in Figure 7. The straightedge was glued on one side of an aluminum block with Duco cement, which is a relatively weak bonding adhesive. Also the soft and flaky texture on the boundary of the fault zone on the retrieved rock sample fragments indicates the weakness of the material inside the fault zone. The minimum local strain in the bulged zone can be estimated from Figure 13 to be $\sim 0.15 \mathrm{~mm} / 24.5 \mathrm{~mm}=0.6 \times$
$10^{-2}$, where $0.15 \mathrm{~mm}$ is the slit width ahead of the bulged zone when the bulge touched the straightedge and $24.5 \mathrm{~mm}$ is the original rock sample diameter.

On the basis of these evidences we infer what might have happened during this test as the following: We have at first a period of microcrack closure, following by a region of linearly elastic behavior. Then we have a period of microcracks opening up uniformly throughout the rock sample. (These behaviors are consistent with lateral strain-stress data in Figure 12.) At about $92 \%$ of the failure load, stress concentration at sample-load block interfaces (probably due to a slight misalignment of loading blocks) caused the microcracks to interact and to coalesce into locally weak zones. These locally weak zones were deformed more readily than the rest of the rock 

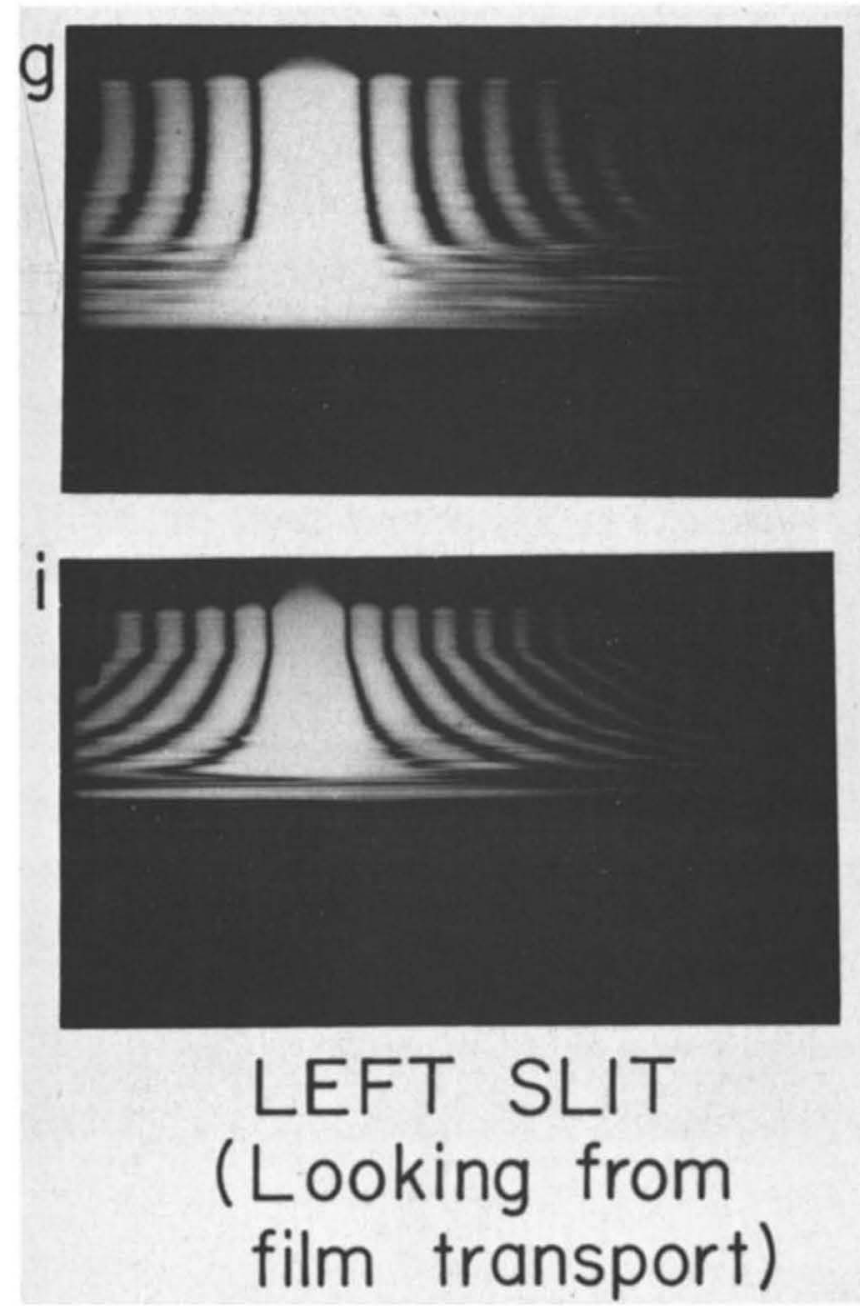

Fig. 7. (continued)

sample because of its reduced deformation moduli, and the locally accentuated strains formed the bulges as observed in the diffraction patterns. These weak zones spread from corners of the rock sample and eventually intersect with each other to cause the failure of the rock sample.

\section{Precursory Bulging Recorded by a Motion Picture Camera}

Because of the large amplitude of the strain inhomogeneity prior to failure recorded by the slit diffraction method in the first test we thought it possible to record the precursory bulging without special magnification. This would permit us to have direct observation of the dynamic fracture process and to use an instrument having a faster frame rate.

Experimental arrangement. The experimental arrangement is similar to the arrangements of the slit diffraction experiment except that the rock sample and the beryllium loading blocks are larger. (The rock sample is $2.9197 \mathrm{~cm}$ in diameter and $6.678 \mathrm{~cm}$ long, and the end loading blocks measure $2.917 \mathrm{~cm}$ in diameter and $3.797 \mathrm{~cm}$ long. Both the rock sample and the end loading blocks have the same finish as described in the slit diffraction experiment.) A piece of ground glass is put between the laser beam and rock sample, so that the rock sample is illuminated by a diffused light source. The distance between the straightedges and the rock sample is also increased to $\sim 0.75 \mathrm{~mm}$. The straightedges are used only for visual reference. The terminal stage of the test is recorded by a $16-\mathrm{mm}$
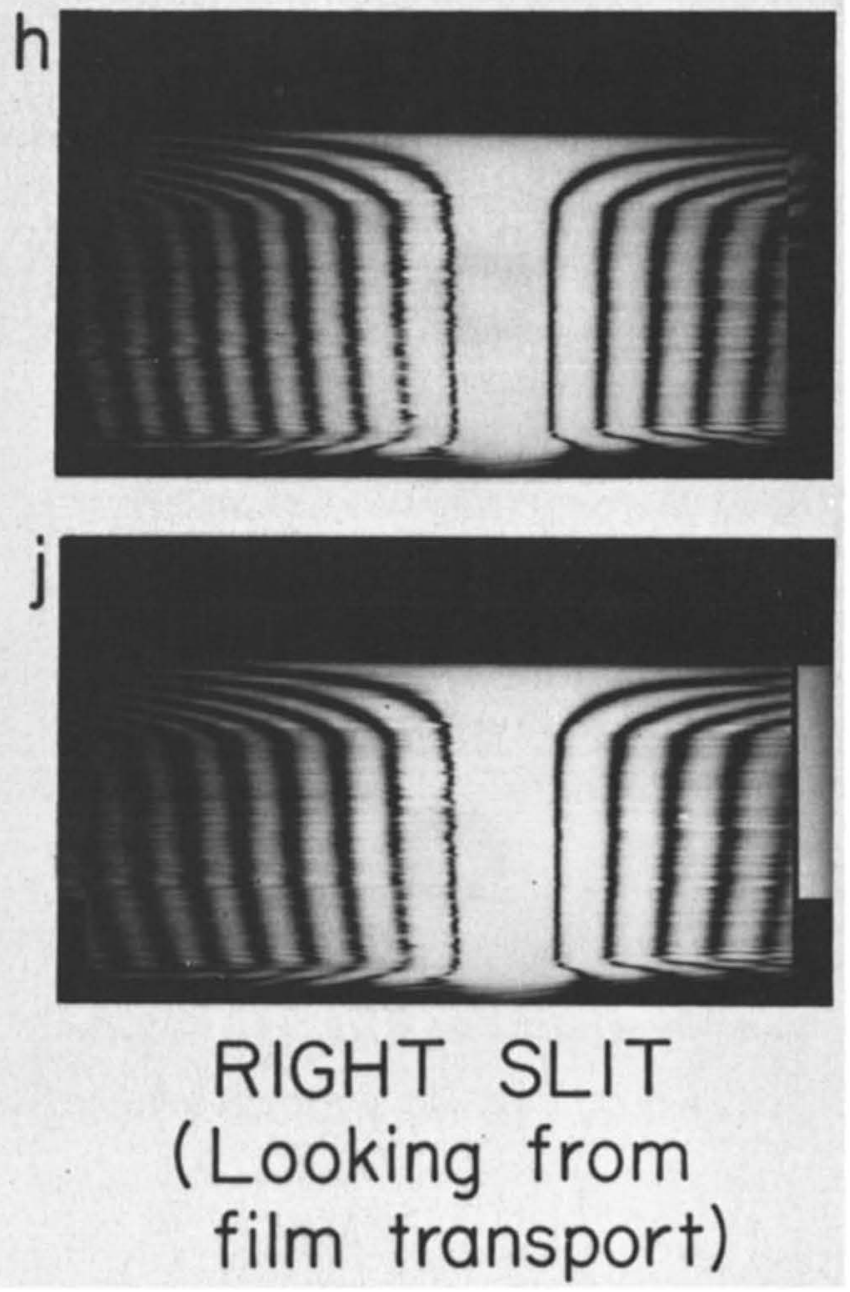

movie camera looking directly toward the sample against the direction of the light beam and focused on the slits formed between the rock sample and the two straightedges.

Results. The motion picture camera was started at a load of $15,263 \mathrm{~kg}$ (2.24-kbar stress). The load was increased steadily until rock sample failure at a load of $16,834 \mathrm{~kg}$ (corresponding to a uniaxial compressive strength of $2.47 \mathrm{kbar}$ ). The time duration between the two loads is $135 \mathrm{~s}$. A series of frames from the film is shown in Figure 15 with the time in seconds counted back from failure marked underneath each frame. The bulge is distinctly visible in Figure 15a. Microcracking activities were generally audible during this stage of the test. Figures $15 a-15 h$ trace the development of this bulge to the beginning of the fracture process. The last six frames before failure are shown in Figures $16 a-16 f$. Figure $16 \mathrm{~g}$ shows the fracture, and Figure $16 h$ is the frame immediately following the fracture. Figures $16 a-16 d$ and $16 f$ all show material being squirted out from the bulged zone. Figures $16 \mathrm{~g}$ and $16 \mathrm{~h}$ show that the main fracture zone coincides with the precursory bulge. This series of photographs demonstrated the formation of a concentrated weak zone as a result of the interaction and coalescence among the microcracks, which then develop into the plane of failure. The bulging is the result of accentuated deformation in the weak zone because of its reduced deformation properties. Again, the strain inhomogeneity is greater than $0.75 \mathrm{~mm} / 29.2 \mathrm{~mm}=2.6 \times 10^{-2}$ in the bulged zone. One important difference, however, exists between the two test 

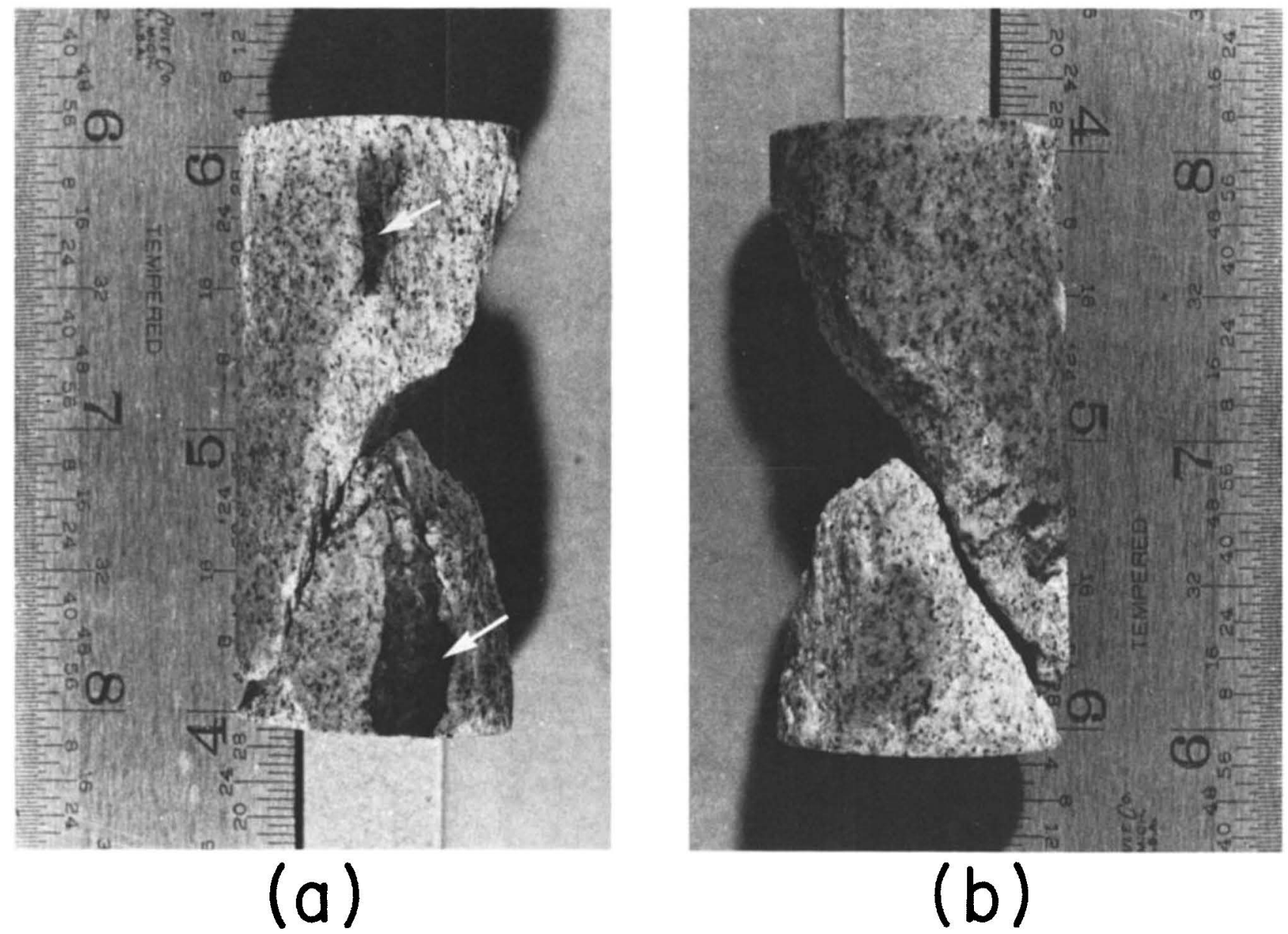

Fig. 8. Rock sample as retrieved from after the experiment. (a) Side facing toward the incident laser beam. Arrow indicates streak of paint painted before the experiment for fault orientation identification. $(b)$ Side of rock sample facing toward the film magazine.

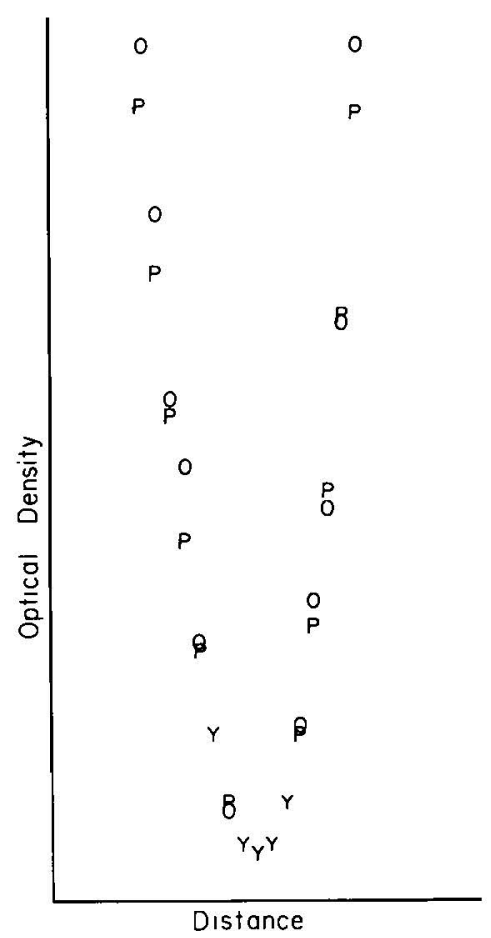

Fig. 9. Typical theoretical fit of intensity near the first intensity minima according to (3). Here, $O$ means observed value, $P$ means theoretically predicted value, and $Y$ means coincidence of $O$ and $P$. Scales are arbitrary. results. The bulging in the first test appeared much earlier ( 9 min before failure) and at a much lower stress level ( $\sim 92 \%$ of the failure strength) than in the second test $(\sim 3.8 \mathrm{~s}$ and $99.7 \%$ of failure strength). The bulging at the corners in the first test was initiated by the stress concentration at the sample-load block interface. This probably is due to a slight misalignment of the beryllium copper loading blocks with respect to the sample. The lateness in appearance and the location of the bulging in the second test indicate a more perfect alignment and a nearly homogeneous stress distribution. The slight inhomogeneity in material properties inside the sample, an inevitable condition inside rocks, causes the stress concentration and the coalescence of microcracks inside the rock sample which precede failure. The second test is therefore relatively independent of the loading boundary conditions and can be considered as an intrinsic property of the rock sample. As a consequence of the manner of the initiation of the bulged zone, the first test shows a single fracture plane, whereas the second test shows a multiple fracture pattern.

\section{Discussion}

We have employed two optical methods to observe the surface deformations of uniaxially compressed rock samples. The method of taking motion pictures is commonplace. The basis of the slit diffraction method, the slit diffraction phenomenon, has been well known since the nineteenth century. Pryor et al. [1972] suggested using slit diffraction for displacement measurement by the diffracted intensity formula for a straight 


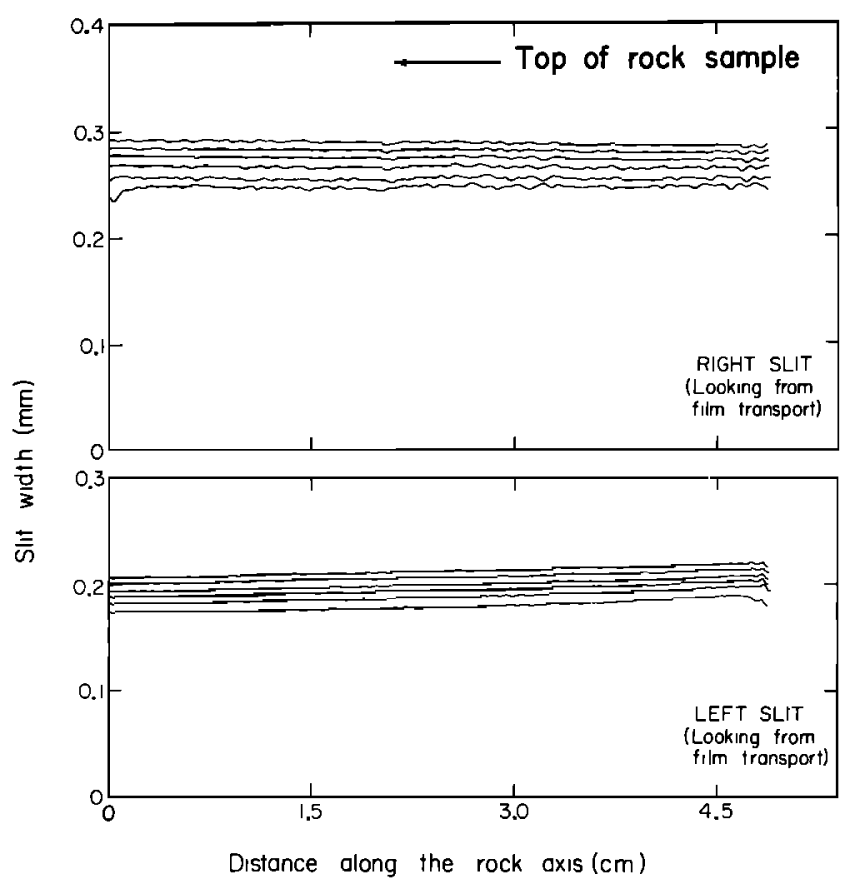

Fig. 10. Slit with data of the two slits in the region of nearly uniform strain throughout the sample. The top figure shows one slit width at different loads, and the bottom figure shows the other slit width at the corresponding loads. The loads are, from the top, 1,790.7 kg (0.37-kbar stress), 3,581.4 kg (0.74-kbar stress), 5,372.1 kg (1.12kbar stress), 7,162.8 kg (1.49-kbar stress), 8,953.5 kg (1.86-kbar stress), 10,296.5 kg (2.14-kbar stress).

slit, (2), without considering the restrictions on the observation angle, the radius of curvature of the diffracting object, and the magnitude of perturbation that can be superimposed on the straight slit. The investigation of radio waves diffracted by hills made by Rice [1953] can be applied to give the conditions for the first two questions, and we have presented in this paper the conditions for the third question.

There are other optical methods for strain measurements. Two of them are the moiré fringe method and holographic

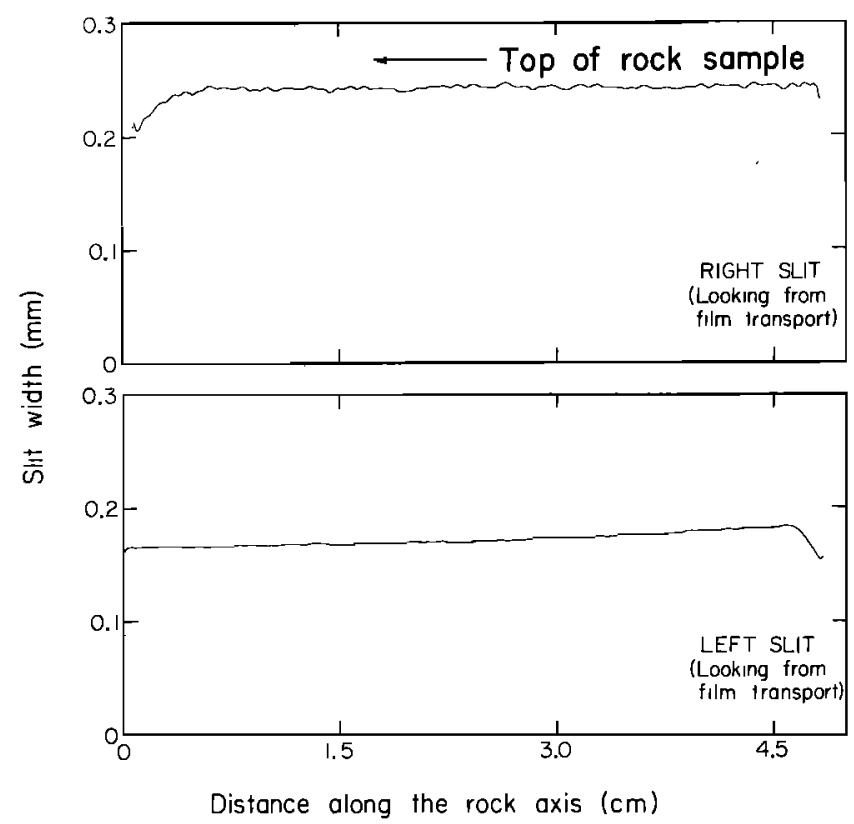

Fig. 11. Slit width data showing onset of strain inhomogeneity. The load is $10,744 \mathrm{~kg}$ (2.23-kbar stress).

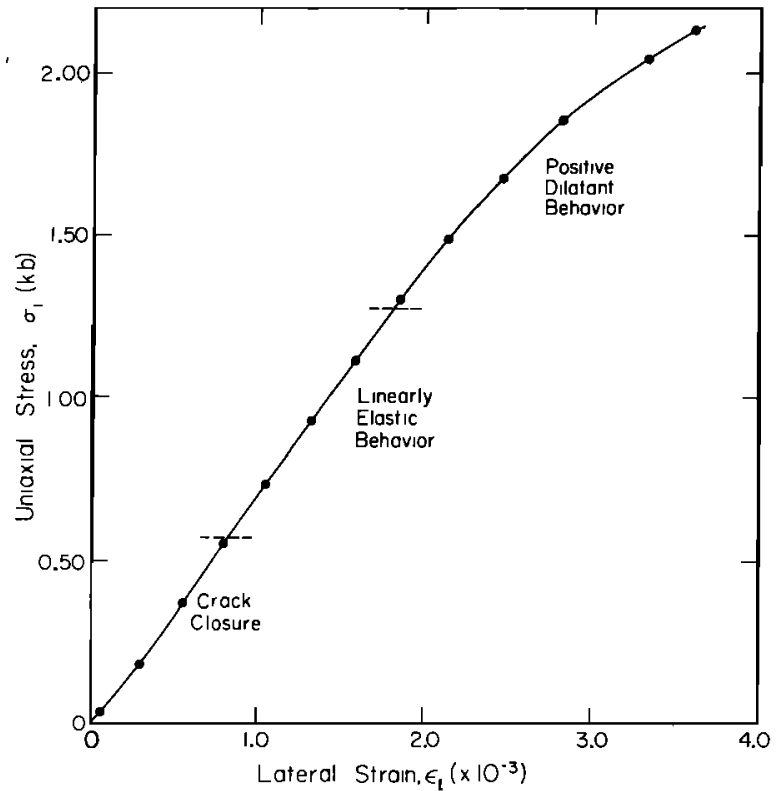

Fig. 12. Lateral strain defined as fractional change in rock sample cross section diameter as a function of uniaxial stress. The figure includes only data in the region of homogeneous strain.

interferometry. Theocaris [1966] gives a comprehensive survey of the moire fringe method, and Collier et al. [1971], for example, give an account of the double-exposure holographic interferometry. The main advantage of the slit diffraction method over these two methods is that the diffracted light from the slit can be arranged to concentrate in a relatively narrow angle and still retain an adequate degree of magnification. This puts a less stringent requirement on the intensity of the light source and implies a relatively short exposure time, or equivalently, a higher time resolution. The concentration of diffracted light in a narrow angle would also make the slit diffraction method more easily adaptable to strain measurement of rock samples under confining pressure and high temperature in an optical triaxial cell.

An additional advantage of the slit diffraction method over holographic interferometry lies in its insensitivity to the microstructure of the rock sample surface, which is expected to be disturbed appreciably in the fault zone near failure. This important advantage enables the slit diffraction method to study the deformation in the fault zone in the final stages of rock fracture. The relatively minor disadvantage of the slit diffraction method is that it can map only one cross section of the rock sample. This can be alleviated by using several pairs of straightedges evenly distributed around the rock sample circumference.

There have been earlier work either locating incipient fracture zones or studying strain concentration before failure. Scholz [1968b] located the microfracture events in a uniaxial compressive test by arrivals of $S$ waves detected with a multitransducer array. He reported a coalescence of microfracturing onto a fracture plane above $92 \%$ of the failure stress. Spetzler et al. [1974] employed double-exposure holographic interferometry to detect dilatation concentration of a cylindrical Westerly granite sample under a uniaxial compressive creep test. They reported a strain concentration early in the creep test (resulting in a bulge growth of $0.48 \mu \mathrm{m}$ in a sample $1.27 \mathrm{~cm}$ in diameter in $320 \mathrm{~s}$ ) but did not give an absolute magnitude of the local strain near failure. Brady [1974] stressed rock samples close to failure, released the load, and 

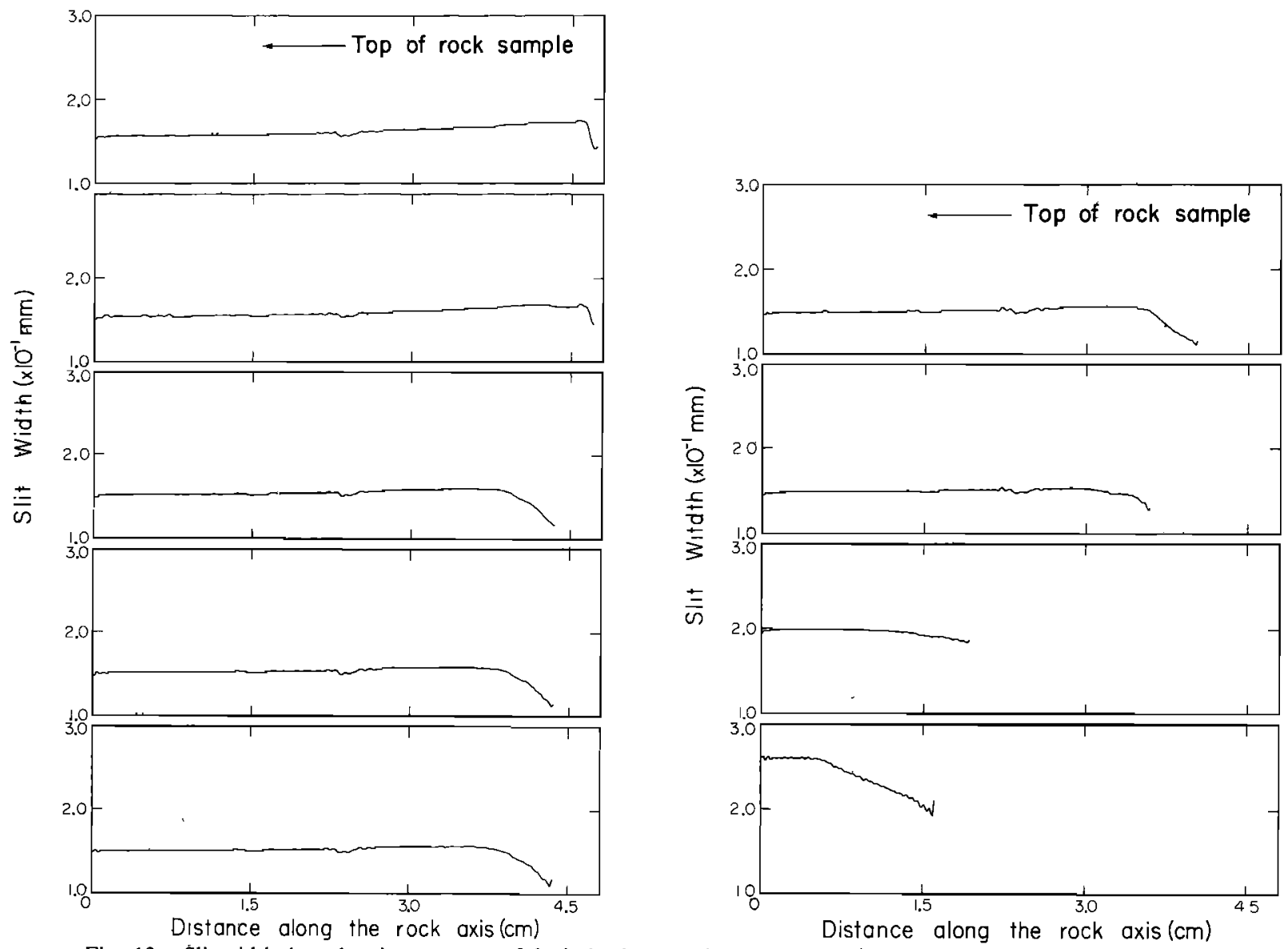

Fig. 13. Slit width data showing progress of the bulged zone. These are deduced from the last nine diffraction patterns of the slit, which belongs to the left side, of the rock looking from film transport. Figures $7 a, 7 c, 7 e, 7 \mathrm{~g}$, and $7 i$ show the last five of these nine diffraction patterns.

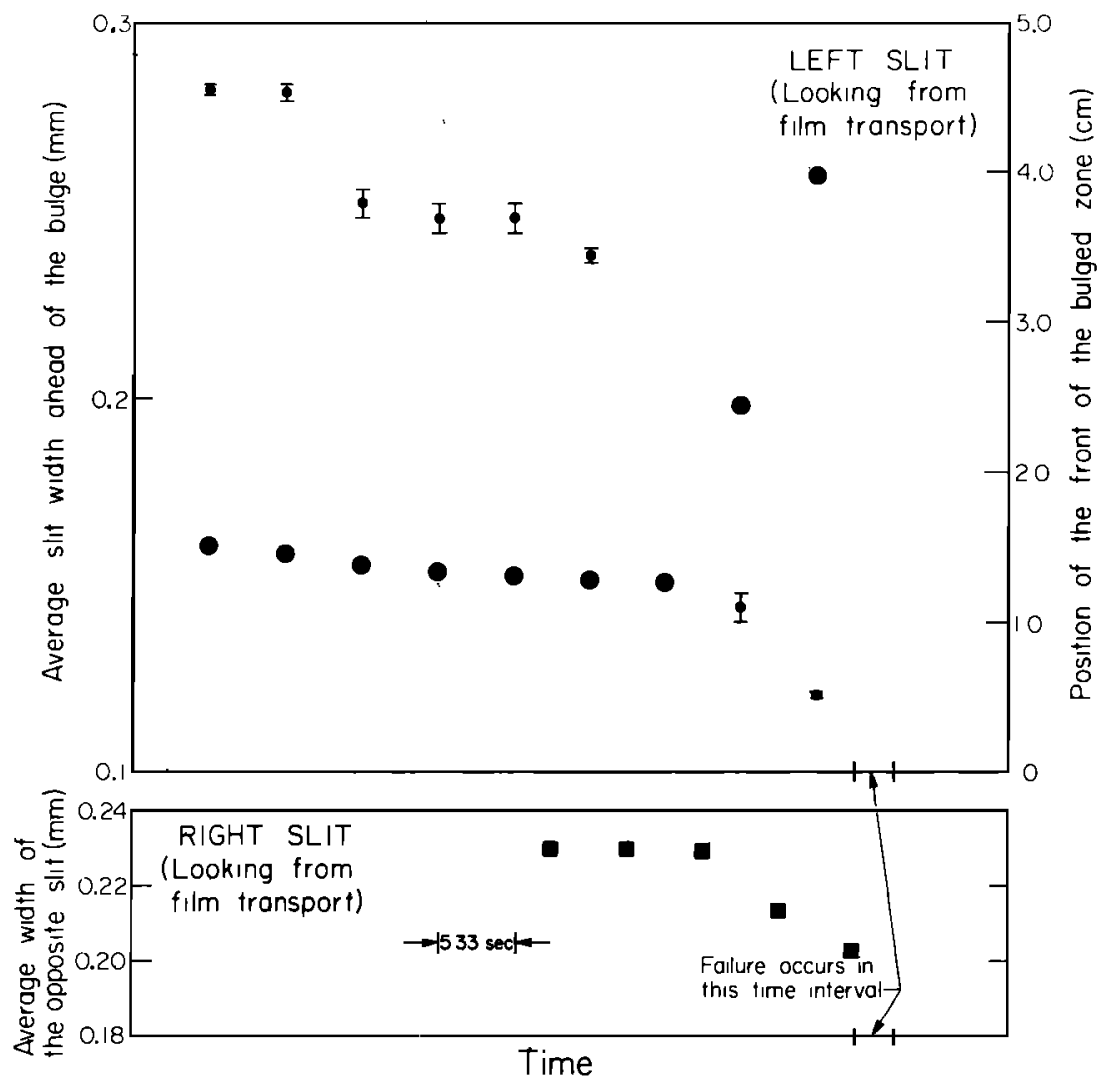

Fig. 14. Average slit width outside of the bulged zone (large dot and solid square) and position of the front of the bulged zone of the slit shown in Figure 13 (small dot). 
then cut the rock samples parallel to the core axis, polished them, and impregnated them with a fluorescent dye to delineate zones of extensive microfracturing during loading. He observed concentrated zones of microfracturing prior to failure both in marbie and in granite. Hadley [1976] studied azimuthal variations of dilatancy by bonding four pairs of strain gages at mid-section of copper-jacketed samples of Westerly granite. Strain anisotropy was noted after the onset of dilatancy, and the major axis of the strain ellipse near failure is nearly perpendicular to the ultimate failure trace. The reported magnitude of the strain anisotropy of the strain ellipse is roughly a factor of 2 . This small amount of strain anisotropy perhaps reflects the limited sampling locations of the strain gage method, as was commented on in the introduction of this paper.

The evidence of the formation of a concentrated weak zone before failure in laboratory testing of rock samples can readily be understood and appreoiated if one reflects on the fact that siress concentration in such a natural granular material is always likely to arise either because of loading configuration or because of material inhomogeneity ipperent in the sample.

The contribution of the present work is the measurement of the magnitude and the precursor time of the local strain inhomogeneity associated $w \mid t h$ the fractupe process. Whitcomb et al. [1973] noted that an empirical relationship exists between the precursor time interval and the earthquake characteristic linear dimension, and Brady [1974] extended this empirical relationship to include data from rock burst and coal mine roof fall. This empirical relationship would predict a precursor time of the order of milliseconds for laboratory rock tests if we adopt the maximum fault dimension to be the largest dimension of the rock sample. The precurapr times of both tests reported in this paper do not fit into this empirical relationship. They are too long by 3 orders of magnitude in comparison with those given by the empirical relationship. The precursor time therefore seems to depend on the loading configuration, local inhomogeneities within the rock sample, and detailed boundary loading conditions, and a simple empirical relationship between preqursor tim and linear fault dịmension may not hold in general for fraptupe events in rock masses.

\section{Appendix; Derivation of (10)}

Denote

$$
\begin{aligned}
\mathscr{g}\left(x_{0}, y_{0}\right) & =\int_{-a / 2}^{+a / 2} f\left(y_{1}\right) \operatorname{sinc}\left(\frac{f\left(y_{1}\right) x_{0}}{\tilde{\lambda} z}\right) \\
& \cdot \exp \left\{i \frac{k}{2 z}\left[\left(y_{1}-y_{0}\right)^{2}-x_{0} f\left(y_{1}\right)\right]\right\} d y_{1}
\end{aligned}
$$

The integral (Al) can be evaluated by the method of stationary phase [e.g., Carrier et al., 1966]. The method of stationary phase makes the following assumptions.

1. Rapid oscillation of the phase occurs except at the stationary point; or equivalently, $k / 2 z$ in (A1) is a real, large number $k / 2 z=235 \mathrm{~cm}^{-2}$ in the present experiment.

2. The function $h\left(y_{1}\right) \equiv\left(y_{1}-y_{2}\right)^{2}-f\left(y_{1}\right) x_{0}$ in (Al) is twice differentiable inside the interval $-a / 2<y_{1}<a / 2$.

3. There exists only one point $\bar{y}$ in the interval $-a / 2<y_{1}$ $<a / 2$ such that $h^{\prime}(\bar{y})=0$. This condition is satisfied if $h^{\prime}\left(y_{1}\right)=$ 0 has a solution and

$$
1-f^{\prime \prime}\left(y_{1}\right) x_{0} / 2 \geq 0 \quad-a / 2<y_{1}<a / 2
$$

If there exists a solution for $h^{\prime}\left(y_{1}\right)=0$ and all of the three conditions above are satisfied, the integral (A1) becomes

$$
\begin{array}{r}
\mathscr{g}\left(x_{0}, y_{0}\right)=f(\bar{y}) \operatorname{sinc}\left(\frac{f(\bar{y}) x_{0}}{\lambda z}\right) \exp \left[i \frac{k}{2 z} h(\bar{y})\right] \\
\cdot \int_{-a / 2}^{+a / 2} \exp \left[i \frac{k}{2 z} \frac{h^{\prime \prime}(\bar{y})}{2}\left(y_{1}-\bar{y}\right)^{2}\right] d y_{1}
\end{array}
$$

plus contribution from the end points.

We will, for the moment, disregard the contribution from the end points. The value $\bar{y}$ in $(10)$ is given by the solution of the equation

$$
\begin{aligned}
\frac{d}{d y_{1}} h\left(y_{1}\right)=\frac{d}{d y_{1}}\left[\left(y_{1}-y_{0}\right)^{2}\right. & \left.-f\left(y_{1}\right) x_{0}\right] \\
& =2\left(y_{1}-y_{0}\right)-f^{\prime}\left(y_{1}\right) x_{0}=0
\end{aligned}
$$

or

$$
\bar{y}-y_{0}=f^{\prime}(\bar{y})\left(x_{0} / 2\right)
$$

Substitute

$$
h^{\prime \prime}(\bar{y})=2-f^{\prime \prime}(\bar{y}) x_{0}
$$

in equation (A3) to get

$$
\begin{aligned}
& g\left(x_{0}, y_{0}\right)=f(\bar{y}) \operatorname{sinc}\left(\frac{f(\bar{y}) x_{0}}{\lambda z}\right) \exp \left[i \frac{k}{2 z} k(\bar{y})\right] \\
& \cdot \int_{-a / 2}^{+a / 2} \exp \left\{i \frac{k}{2 z}\left(1-x_{0} \frac{f^{\prime \prime}(\bar{y})}{2}\right)\left(y_{1}-\bar{y}\right)^{2}\right\} d y_{1}
\end{aligned}
$$

Assume further that

$$
\begin{aligned}
\left|f^{\prime}\left(y_{1}\right)\left(x_{0} / 2\right)\right| \leq & a / N \quad-a / 2<y_{1}<a / 2 \\
& -a\left[\frac{1}{2}+(1 / N)\right]<y_{p}<a\left[\frac{1}{2}+(1 / N)\right]
\end{aligned}
$$

Equation (A4) implies

$$
\left|\bar{y}-y_{0}\right| \leq a / N
$$

i.e., $\bar{y}=y_{0}$ to an accuracy within $a / N$. Equation (A5) becomes

$$
\begin{gathered}
g\left(x_{0}, y_{0}\right)=f\left(y_{0}\right) \operatorname{sinc}\left(\frac{f\left(y_{0}\right) x_{0}}{\dot{\lambda} z}\right) \exp \left[i \frac{k}{2 z} h\left(y_{0}\right)\right] \\
\cdot \int_{-a / 2}^{+a / 2} \exp \left\{i \frac{k}{2 z}\left[1-\frac{f^{\prime \prime}\left(y_{0}\right) x_{0}}{2}\right]\left(y_{1}-y_{0}\right)^{2}\right\} d y_{1}
\end{gathered}
$$

If further

$$
\left|f^{\prime \prime}\left(y_{0}\right) x_{0} / 2\right| \ll 1
$$

then the integral $\mathscr{G}\left(x_{0}, y_{0}\right)$ becomes

$$
\begin{aligned}
g\left(x_{0}, y_{0}\right)= & f\left(y_{0}\right) \operatorname{sinc}\left(\frac{f\left(y_{0}\right) x_{0}}{\lambda z}\right) \exp \left[i \frac{k}{2 z} h\left(y_{0}\right)\right] \\
& \cdot \int_{-a / 2}^{+a / 2} \exp \left[i \frac{k}{2 z}\left(y_{1}-y_{0}\right)^{2}\right] d y_{1} \\
= & f\left(y_{0}\right) \operatorname{sinc}\left(\frac{f\left(y_{0}\right) x_{0}}{\lambda z}\right) \exp \left[i \frac{k}{2 z} h\left(y_{0}\right)\right]\left(\frac{\pi z}{k}\right)^{1 / 2} \\
& \cdot\left\{C\left(\eta_{2}\right)-C\left(\eta_{1}\right)+i\left[S\left(\eta_{2}\right)-S\left(\eta_{1}\right)\right]\right\}
\end{aligned}
$$



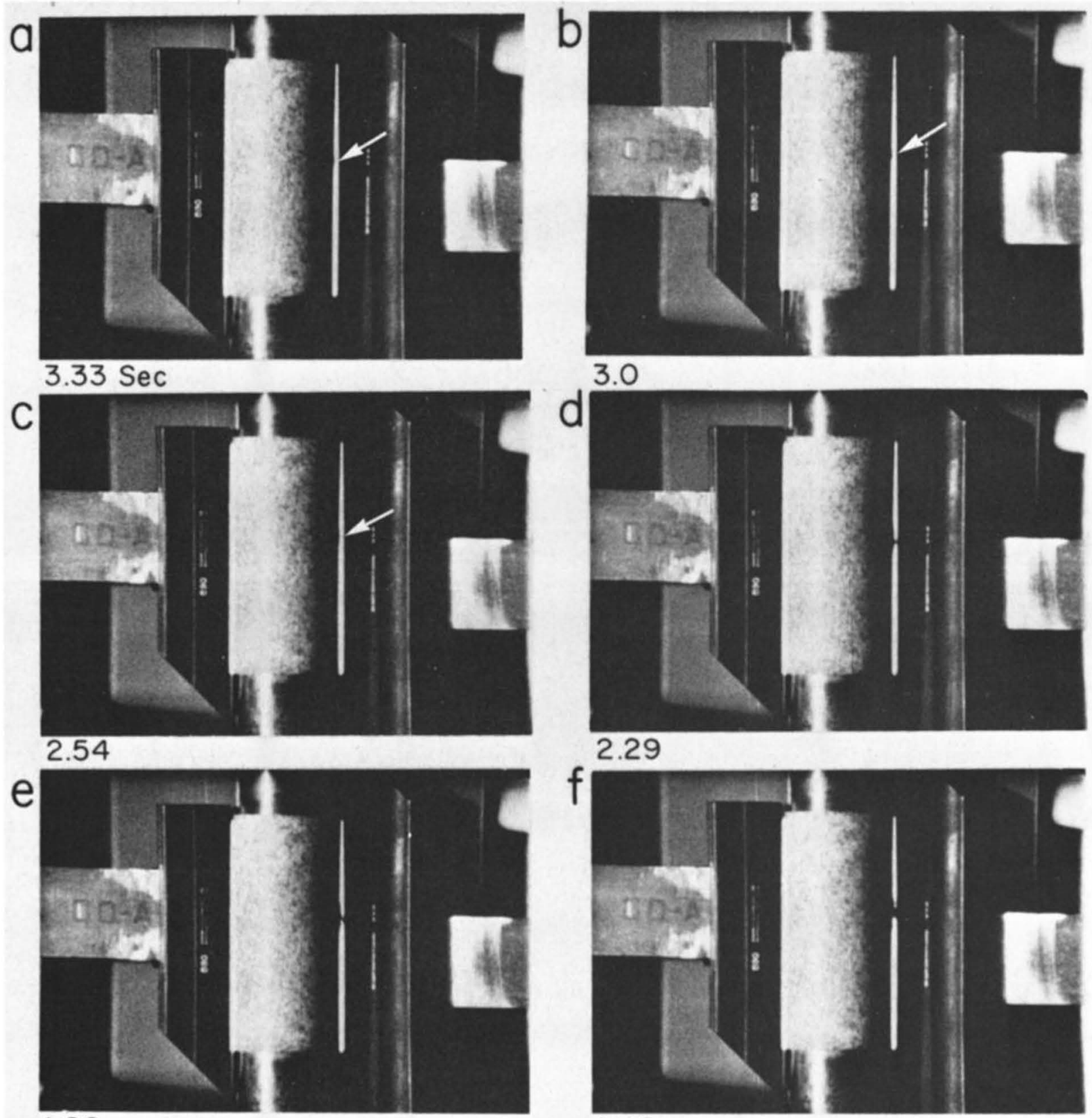

\subsection{8}

\subsection{6}

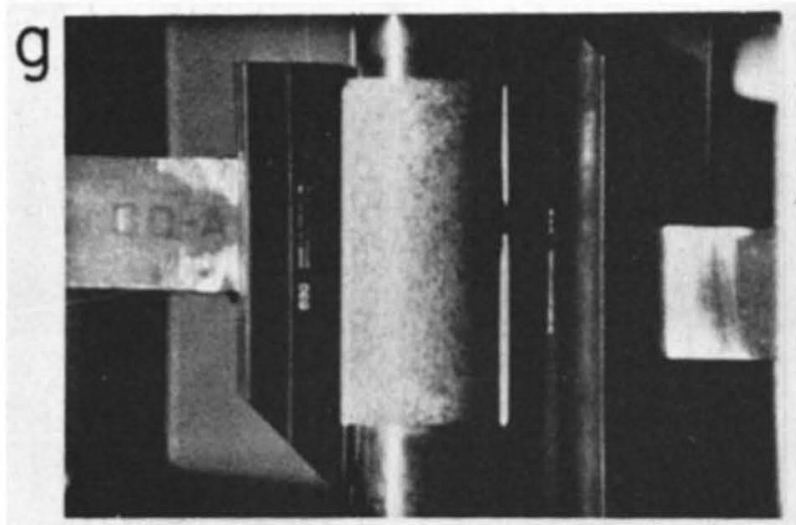

$1.04 \mathrm{Sec}$

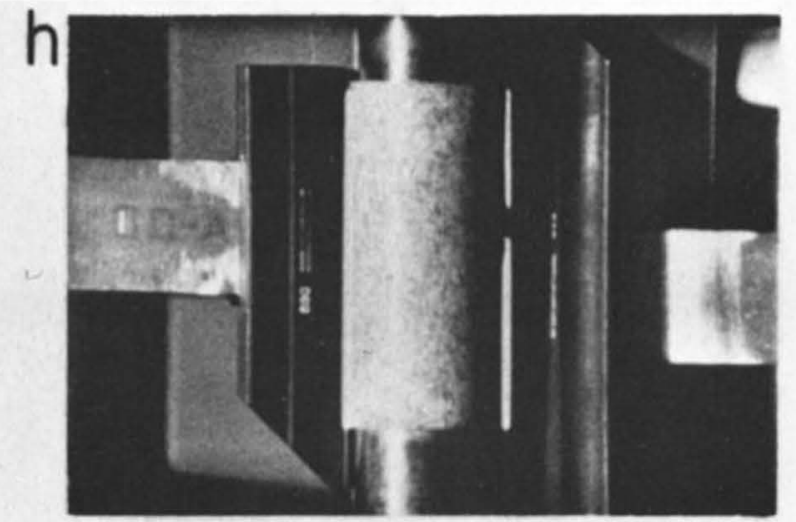

0.45

Fig. 15. Series of photographs showing onset of bulge in the middle of the rock sample. The time (in seconds) before failure is listed under each photograph. (Copyright WGBH Educational Foundation.) 

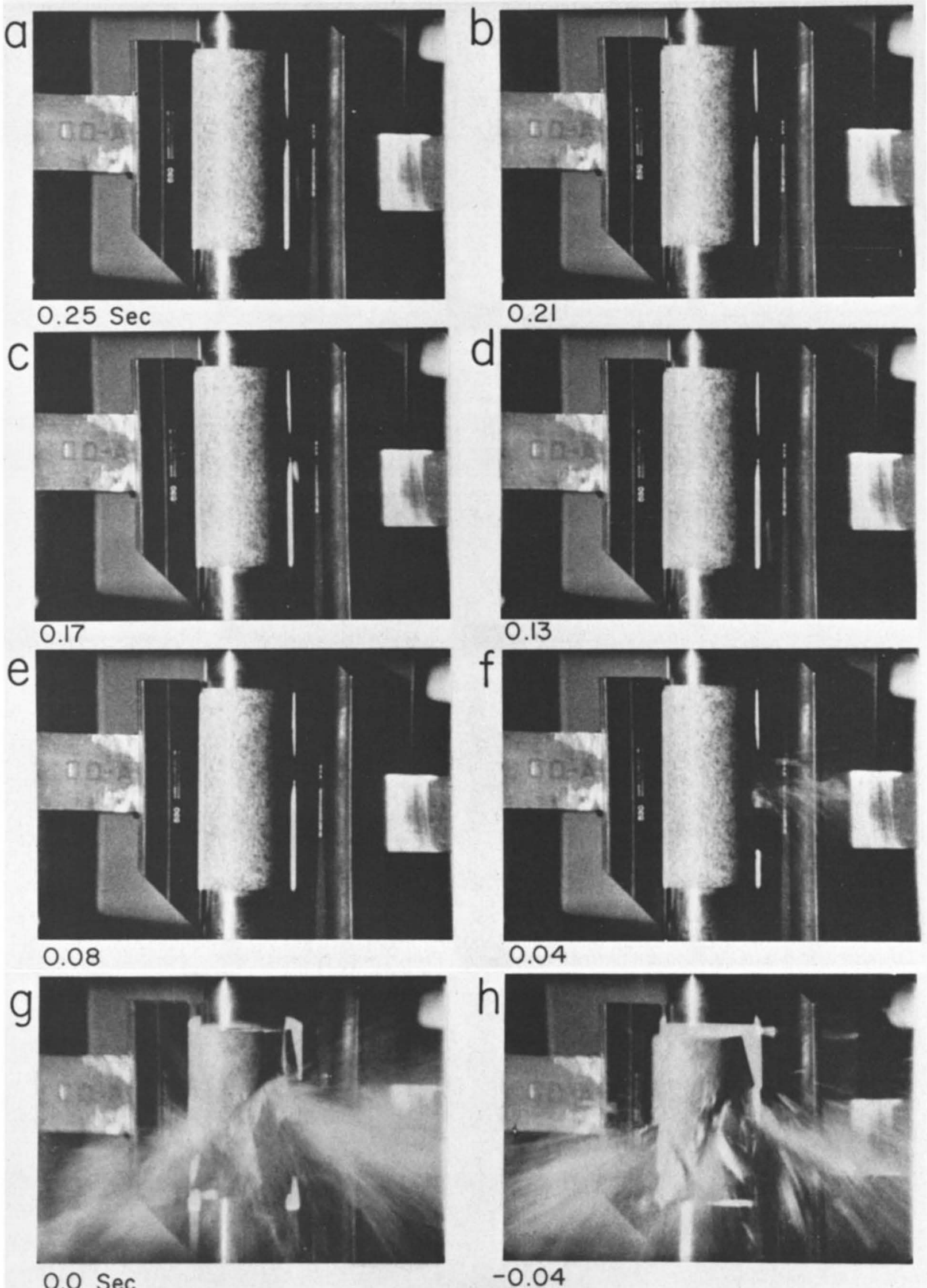

$0.0 \mathrm{Sec}$

$$
-0.04
$$

Fig. 16. Series of photographs showing fracture of rock sample. The time (in seconds) before failure is listed under each photograph. (Copyright WGBH Educational Foundation.) 
where

$$
C(\eta)=\int_{0}^{\eta} \cos \left(\frac{\pi t^{2}}{2}\right) d t \quad S(\eta)=\int_{0}^{\pi} \sin \left(\frac{\pi t^{2}}{2}\right) d t
$$

are the Fresnel integrals, and

$$
\eta_{1}=-(k / \pi z)^{1 / 2}\left[(a / 2)+y_{0}\right] \quad \eta_{2}=(k / \pi z)^{1 / 2}\left[(a / 2)-y_{0}\right]
$$

The contribution to the integral from the end points in (A3) is given by

$$
-\frac{\exp [i(k / 2 z) h(-a / 2)]}{(k / 2 z)\left|h^{\prime}(-a / 2)\right|}+\frac{i \exp [i(k / 2 z) h(a / 2)]}{(k / 2 z) h^{\prime}(a / 2)}
$$

This is of the order $O\left[(k / 2 z)^{-1 / 2}\right]$ in comparison with the righthand side of (A10), and $k / 2 z$ is assumed to be a large number in the stationary phase approximation.

The amplitude of the diffracted wave, (7), is therefore

$$
\begin{gathered}
U\left(x_{0}, y_{0}\right)=i \frac{e^{i k z}}{\lambda z} \exp \left[\frac{i k x_{0}{ }^{2}}{2 z}\right] \\
\cdot \exp \left[i \frac{k}{2 z} h\left(y_{0}\right)\right] \operatorname{sinc}\left[\left(\frac{f\left(y_{0}\right) x_{0}}{\lambda z}\right)\right]\left(\frac{\pi z}{k}\right)^{1 / 2} \\
\cdot\left\{C\left(\eta_{2}\right)-C\left(\eta_{1}\right)+i\left[S\left(\eta_{2}\right)-S\left(\eta_{1}\right)\right]\right\}
\end{gathered}
$$

which gives the intensity distribution expressed by (10).

Acknowledgments. The authors wish to thank W. G. Knauss and C. D. Babcock, Jr., for providing the use of a Baldwin testing machine and R. F. Scott, Don L. Anderson, J. H. Whitcomb, and N. George for their interest and some discussions concerning this work. We also wish to thank E. Steffensen and N. O. B. Motta for their valuable technical assistance. The motion picture was taken by a crew under the direction of J. Angier from WGBH Educational Foundation, Boston. This work was supported by the Jet Propulsion Laboratory/NASA under contract 64597 and was supported partially by the Air Force Office of Scientific Research under AFOSR 72-2234. Contribution 2673, Division of Geological and Planetary Sciences, California Institute of Technology, Pasadena, California.

\section{REFERENCES}

Aggarwal, Y. P., L. R. Sykes, J. Armbruster, and M. L. Sbar, Premonitory changes in seismic velocities and prediction of earthquakes, Nature, 241, 101-104, 1973.

Anderson, D. L., and J. H. Whitcomb, The dilatancy-diffusion model of earthquake prediction, Proceedings of the Conference on Tectonic Problems of the San Andreas Fault System, Stanford Univ. Publ. Geol. Sci., 13, 417-426, 1973.

Barnett, J. D., and F. S. Harris, Jr., Test of the effect of edge parameters on small-angle Fresnel diffraction of light at a straight edge, $J$. Opt. Soc. Amer., 52, 637-643, 1962.

Beaumont, C., and J. Berger, Earthquake prediction: Modification of the earth tide tilts and strains by dilatancy, Geophys. J. Roy. Astron. Soc., 39, 111-121, 1974.

Brace, W. F., and R. J. Martin III, A test of the law of effective stress for crystalline rocks of low porosity, Int. J. Rock Mech. Miner. Sci., 5, 415-426, 1968 .

Brace, W. F., and A. S. Orange, Electrical resistivity changes in saturated rocks during fracture and frictional sliding, $J$. Geophys. Res., 73, 1433-1445, 1968.

Brace, W. F., B. W. Paulding, Jr., and C. Scholz, Dilatancy in the fracture of crystalline rocks, J. Geophys, Res., 7I, 3939-3953, 1966.

Brady, B. T., Theory of earthquakes, 1, A scale independent theory of rock failure, Pure Appl. Geophys., II2, 701-725, 1974.

Bridgman, P., Volume changes in the plastıc stages of simple compression, J. Appl. Phys., 20, 1241-1251, 1949.
Carrier, G. F., N. Krook, and C. E. Pearson, Functions of a Complex Variable, pp. 272-275, McGraw-Hill, New York, 1966.

Collier, R. J., C. B. Burckhardt, and L. H. Lin, Optical Holography, pp. 418-453, Academic, New York, 1971.

Fai, W-C, Dynamic pulse method of determining elastic parameters of rock samples under high all-sided pressures, Bull. Acad. Sci. USSR Geophys. Ser., 10, 1004-1008, 1961.

Goodman, J. W., Introduction to Fourier Optics, pp. 58-59, 63, McGraw-Hill, New York, 1968.

Hadley, K., Azimuthal variation of dilatancy, J. Geophys. Res., 80 , 4845-4850, 1975.

Handin, J., R. V. Hager, Jr., M. Friedman, and J. N. Feather, Experimental deformation of sedimentary rocks under confining pressure: Pore pressure tests, Bull. Amer. Assoc. Petrol. Geol., 47, 717-755, 1963.

Knopoff, L., Seismic wave velocities in Westerly granite, Trans. $A G U$, 35, 969-972, 1954.

Marchand, E. W., and E. Wolf, Consistent formulation of Kirchhoff's diffraction theory, J. Opt. Soc. Amer., 56, 1712-1722, 1966.

Matsushima, S., On the flow and fracture of igneous rocks, Disaster Prev. Res. Inst. Kyoto Univ. Bull., 36, 2-9, 1960a.

Matsushima, S., On the deformation and fracture of granite under high confining pressure, Disaster Prev. Res. Inst. Kyoto Univ. Bull., 36, 11-20, $1960 b$.

Matsushima, S., Variation of the elastic wave velocities of rocks in the process of deformation and fracture under high pressure, Disaster Prev. Res. Inst. Kyoto Univ. Bull., 32, 2-8, 1960c.

Nur, A., Dilatancy, pore fluids and premonitory variations of $t_{s} / t_{p}$ travel times, Bull. Seismol. Soc. Amer., 62, 1217-1222, 1972.

Oberg, E., and F. D. Jones, Machinery's Handbook, 16th ed., p. 500, Industrial, New York, 1962.

Pearson, J. E., T. C. McGill, S. Kurtin, and A. Yariv, Diffraction of Gaussian laser beams by a semi-infinite plane, J. Opt. Soc. Amer., 59, 1440-1445, 1969.

Pryor, T. R., O. L. Hageniers, and W. P. T. North, Diffractographic dimensional measurement, 1, Displacement measurement, Appl. Opt., II, 308-313, 1972.

Reynolds, O., Experiments showing dilatancy, a property of granular material, possibly connected with gravitation, Sci. Pap., 2, 217-227, Cambridge University Press, New York, 1901.

Rice, S. O., Diffraction of plane radio waves by a parabolic cylinder, calculation of shadows behind hills, Bell Syst. Tech. J., 33, 417-504, 1953.

Scholz, C. H., Microfracturing and the inelastic deformation of rock in compression, $J$. Geophys. Res.. 73, 1417-1432, 1968 a.

Scholz, C. H., Experimental study of the fracturing process in brittle rock, J. Geophys. Res., 73, 1447-1454, 1968b. (Correction, J. Geophys. Res., 73, 4794, 1968.)

Scholz, C. H., L. R. Sykes, Y. P. Aggarwal, Earthquake prediction: A physical basis, Science, 181, 803-810, 1973.

Silver, S., Microwave aperture antennas and diffraction theory, $J$. Opt. Soc. Amer., 52, 131-139, 1962.

Simmons, G., and W. F. Brace, Comparison of static and dynamic measurements of compressibility of rocks, J. Geophys. Res., 70. 5649-5656, 1965.

Spetzler, H., C. H. Scholz, and C.P Lu, Strain and creep measurement on rocks by holographic interferometry, Pure Appl. Geophys., II2, 571-581, 1974.

Theocaris, P. S., Moiré fringes: A powerful measuring device, in Applied Mechanics Surveys, edited by H. N. Abramson, H. Liebowitz, J. M. Crowley, and S. Juhasz, pp. 613-626, Spartan, Washington, D. C., 1966.

Whitcomb, J. H., New vertical geodetics, J. Geophys. Res., in press, 1976.

Whitcomb, J. H., J. D. Garmany, and D. L. Anderson, Earthquake prediction: Variation of seismic velocities before the San Fernando earthquake, Science, 180, 632-635, 1973.

Wolf, E., and E. W. Marchand, Comparison of the Kirchhoff and the Rayleigh-Sommerfeld theories of diffaction at an aperture, $J$. $O p t$. Soc. Amer., 54, 587-594, 1964.

(Received September 15, 1975; revised March 25, 1976; accepted March 25, 1976.) 